\title{
A structural subgrid-scale model for the collision-related statistics of inertial particles in large-eddy simulations of isotropic turbulent flows
}

Cite as: Phys. Fluids 32, 095103 (2020); https://doi.org/10.1063/5.0018756

Submitted: 17 June 2020 . Accepted: 12 August 2020 . Published Online: 01 September 2020

(D) Zhideng Zhou (周志登), (D) Shizhao Wang (王士召), (D) Xiaolei Yang (杨晓雷), and (D Guodong Jin (晋国栋)

\section{ARTICLES YOU MAY BE INTERESTED IN}

Visualizing droplet dispersal for face shields and masks with exhalation valves

Physics of Fluids 32, 091701 (2020); https://doi.org/10.1063/5.0022968

Effects of the collision model in interface-resolved simulations of particle-laden turbulent channel flows

Physics of Fluids 32, 103303 (2020); https://doi.org/10.1063/5.0020995

A vortex identification method based on local fluid rotation

Physics of Fluids 32, 015104 (2020); https://doi.org/10.1063/1.5133815

\section{Physics of Fluids GALLERY OF GOVERS}




\title{
A structural subgrid-scale model for the collision-related statistics of inertial particles in large-eddy simulations of isotropic turbulent flows
}

\author{
Cite as: Phys. Fluids 32, 095103 (2020); doi: 10.1063/5.0018756 \\ Submitted: 17 June 2020 - Accepted: 12 August 2020 • \\ Published Online: 1 September 2020
}

\author{
Zhideng Zhou (周志登), ${ }^{1,2}$ (D) Shizhao Wang (王士召), ${ }^{1,2}$ (D) Xiaolei Yang (杨晓雷), ${ }^{1,2}$ (D) \\ and Guodong Jin (晋国栋) ${ }^{1,2, a)}$ (D)
}

\begin{abstract}
AFFILIATIONS
${ }^{1}$ The State Key Laboratory of Nonlinear Mechanics, Institute of Mechanics, Chinese Academy of Sciences, Beijing 100190, China ${ }^{2}$ School of Engineering Sciences, University of Chinese Academy of Sciences, Beijing 100049, China
\end{abstract}

a) Author to whom correspondence should be addressed: gdjin@lnm.imech.ac.cn

\begin{abstract}
In large-eddy simulations of particle-laden isotropic turbulent flows, the collision of inertial particles is strongly influenced by missing small-scale turbulence. In this paper, we apply the Kinematic Simulation with Approximate Deconvolution (KSAD) model to determine the contribution of small-scale turbulence to the motion of inertial particles and improve the prediction accuracy of the radial distribution function (RDF) and radial relative velocity (RRV), which are closely related to particle collisions. Different values of Stokes numbers (St), which are defined as the ratio of the particle response time to the Kolmogorov time scale, are considered. The KSAD model significantly improves the prediction accuracy of the RRV for all considered St. For the prediction of RDF, good agreement between the KSAD model and direct numerical simulations is only observed for large $S t$, i.e., $S t \geq 2.0$. To explore the reason for the poor prediction of the KSAD model for small $S t$, we compare the Eulerian statistics of the flow fields and the Lagrangian properties of the particles from different simulations and find the key reason is that the Gaussian turbulence generated in the kinematic simulation model is inadequate in recovering the vortex centrifugal effect of small-scale turbulence on the inertial particle clustering at small St.
\end{abstract}

Published under license by AIP Publishing. https://doi.org/10.1063/5.0018756

\section{INTRODUCTION}

Inertial particles exist in many industrial applications and

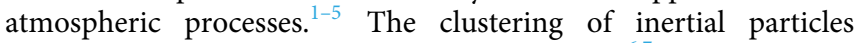
(often referred to as preferential concentration ${ }^{6,7}$ ) can influence a broad range of processes, e.g., particle settling, 8,9 evaporation/condensation, ${ }^{10,11}$ interparticle collisions and coagulation, ${ }^{12-14}$ and the transmission of viruses through a human sneeze. ${ }^{15,16}$ Compared with experiments, numerical simulations can provide data with high temporal and spatial resolutions, which are crucial for studying the mechanism in the aforementioned processes. Computational models for particle-laden turbulence can be classified into three types: direct numerical simulations (DNSs), large-eddy simulations (LESs), and the Reynolds averaged Navier-Stokes (RANS) simulations. DNS, which can resolve all of the turbulent scales and accurately predict the inertial particle statistics, ${ }^{17-19}$ is still not feasible for simulating particle-laden turbulent flows at high Reynolds numbers because of the extremely high computational cost. ${ }^{20,21}$ The computational cost of RANS is low. However, RANS is not able to predict turbulent fluctuations, which are essential in simulating particle statistics. Moreover, LES, which directly simulates large, energy-containing scales and models the effects of subgrid scales (SGSs) on the large-scale processes, is emerging as an important tool for investigating particle-laden turbulent flows. ${ }^{22,23}$ In LESs of single-phase turbulent flows, the subgrid modeling can severely affect the resultant turbulent statistics such as velocity decorrelation time scales and two- or multi-particle relative dispersion of fluid particles. LESs can overpredict the velocity decorrelation time scales ${ }^{24,2}$ 
while underpredicting the relative dispersion of fluid particles. ${ }^{26,27}$ An elliptic model was proposed to correctly characterize the spacetime correlation and dynamic coupling effects in turbulent flows with the second-order approximation. The model can be used as a criterion for evaluating the performance of LESs of turbulent flows. ${ }^{28,29}$ In LESs of particle-laden turbulent flows, missing subgridscale (SGS) turbulence has a strong influence on the collision-related statistics of inertial particles at small and intermediate Stokes numbers. ${ }^{30,31}$ It is a challenging task to develop SGS models that can accurately capture the effects of missing scales on inertial particles. As a first step toward this goal, we test the capability of the Kinematic Simulation with Approximate Deconvolution (KSAD) model, which was developed in our previous work and has been successfully applied to the relative dispersion of fluid particles, ${ }^{32}$ in predicting collision-related statistics.

Subgrid-scale turbulence has profound effects on the collision of inertial particles. Sundaram and Collins ${ }^{17}$ investigated the collision statistics of heavy particles suspended in isotropic turbulence and found that particles are depleted in regions of high vorticity at low Stokes numbers $(S t)$ but are relatively unresponsive to smallscale structures at high St. Following that, Ray and Collins ${ }^{19}$ systematically studied the effect of small-scale turbulence on the radial distribution function (RDF) and radial relative velocity (RRV) of inertial particles by applying a sharp spectral filter to the DNS velocity field; similar to the observations by Fede and Simonin, ${ }^{30}$ they found that the filtering process decreases the RDF at low $S t$ and the RRV at all St but increases the RDF at high St. Kuerten and Vreman $^{33}$ performed DNSs and LESs of particle-laden turbulent channel flows and found that conventional LES methods cannot accurately predict turbophoresis. Marchioli et al. ${ }^{34}$ investigated the influence of subgrid turbulence on particle segregation and accumulation by performing a priori and a posteriori LESs of inertial particle dispersion in turbulent channel flows based on two types of coarse grids; they observed that LES inaccurately predicts the local preferential particle segregation for all grids and noted that subgrid closure models for particles may need information on the high-order moments of velocity fluctuations.

To accurately predict the inertial particle statistics in LESs, a variety of models have been developed to identify the effects of small-scale turbulence on particle motion. The approximate deconvolution model (ADM) proposed by Stolz and Adams ${ }^{35}$ was applied to model the subgrid effects in an LES of a particle-laden turbulent flow. ${ }^{36,37}$ However, although the ADM improved the prediction of the resolved scales near the filter width, it could not recognize the velocity fluctuations of small-scale turbulence, which are important for predicting particle clustering. ${ }^{31,38}$ To compensate for the contribution of subgrid fluctuations on particle motion, stochastic models were constructed, ${ }^{39-41}$ but these models cannot properly simulate the interactions between particles and turbulent structures or the relative motion between particles because the SGS velocity field is treated as white noise. ${ }^{38}$ To remedy this issue, Ray and Collins ${ }^{42}$ used a properly tuned kinematic simulation (KS) a priori to predict the RDF and relative velocity in a filtered DNS (FDNS) of a particleladen isotropic turbulence; they showed that the FDNS+KS model accurately predicts particle clustering when $S t \geq 2.0$. To improve the capability of LESs in predicting preferential concentration in particle-laden turbulent flows, Bassenne et al. ${ }^{43}$ recently proposed a spectrally enriched differential filter (SDF) model based on the dynamic differential filter model; ${ }^{44}$ they observed increased RDF agreement between the SDF model and the DNS, except for inertial particles at $S t \sim 1$. Moreover, the machine learning methods, utilized for data-driven deconvolution, ${ }^{45,46}$ closure modeling, ${ }^{47,48}$ and super-resolution reconstruction of low-resolution flow fields, ${ }^{49,50}$ can be used to account for the effects of SGS turbulence on particle motion.

The KSAD model performs well and significantly improves the prediction accuracy in the LES of the relative dispersion of fluid particles. ${ }^{32}$ However, the interaction between inertial particles and turbulence involves a wider range of scales of turbulent motions due to the inertia. The relative dispersion of inertial particles is much more complex than that of fluid particles, which poses a challenge for LESs of particle-laden turbulence. It is of significance to evaluate the performance of the KSAD model on LESs of relative dispersion of inertial particles. The motivation of this study is to assess the performance of the KSAD model in improving the prediction accuracy in particle statistics and explore its advantages and disadvantages in LESs of inertial particles. The objective is to apply the KSAD model to predict the collision-related statistics of inertial particles specifically in LESs of isotropic turbulent flows and to understand the possible mechanics of inability to predict the preferential concentration at small Stokes numbers. First, we assess the capability of the KSAD model to predict the RDF and RRV at different $S t$ by comparing the results from LESs with the KSAD model (LES+KSAD) with those from DNSs and LESs without the KSAD model. We then compare the Eulerian statistics of the flow fields and the Lagrangian flow properties sampled along the particle paths obtained from the DNS, FDNS, FDNS+KS, LES, and LES+KSAD methods and discuss the mechanisms of particle clustering at low $S t$, for which the KSAD model is generally ineffective in predicting the RDF.

The paper is organized as follows: the employed numerical methods and governing equations are described in Sec. II; the KSAD model is introduced in Sec. III; the ability of the KSAD model to predict the collision-related statistics of inertial particles is evaluated in Sec. IV, and a discussion of the mechanism related to the poor performance of the KSAD model in predicting the RDF at low St is presented; and finally, conclusions are drawn in Sec. V.

\section{NUMERICAL METHODS}

We first describe the governing equations and numerical methods employed in DNSs and LESs in Secs. II A and II B, respectively. We then present the methods employed in the FDNS and the governing equation for the motion of inertial particles in Secs. II $\mathrm{C}$ and II D.

\section{A. Direct numerical simulation}

The Navier-Stokes equation for incompressible flows is

$$
\begin{gathered}
\frac{\partial \mathbf{u}}{\partial t}=\mathbf{u} \times \boldsymbol{\omega}-\nabla\left(\frac{p}{\rho}+\frac{1}{2} \mathbf{u}^{2}\right)+v \nabla^{2} \mathbf{u}+\mathbf{f}(\mathbf{x}, t), \\
\nabla \cdot \mathbf{u}=0,
\end{gathered}
$$

where $\mathbf{u}$ denotes the velocity field; $\boldsymbol{\omega}=\nabla \times \mathbf{u}$ denotes the vorticity field; and $p, \rho$, and $v$ are the pressure, density, and kinematic viscosity of the fluid, respectively. $\mathbf{f}(\mathbf{x}, t)$ is an artificial forcing term to input 
energy into the flow and maintain the level of turbulence. The setting of $\mathbf{f}(\mathbf{x}, t)$ will be implemented in Fourier space and explained in the following context.

A DNS of homogeneous and isotropic turbulence was performed using a standard pseudo-spectral method in a periodic cubic flow domain with each edge length $L=2 \pi$. Additionally, the domain was discretized uniformly into $N^{3}$ grids. In Fourier space, Eqs. (1) and (2) can be represented as $\left(k \leq k_{\max }\right)$

$$
\left(\frac{\partial}{\partial t}+v k^{2}\right) \hat{\mathbf{u}}(\mathbf{k}, t)=\mathbf{P}(\mathbf{k}) \mathcal{F}(\mathbf{u} \times \boldsymbol{\omega})+\hat{\mathbf{f}}(\mathbf{k}, t),
$$

where $\hat{\mathbf{u}}(\mathbf{k}, t)$ denotes the Fourier coefficient or the fluid velocity in Fourier space and $\mathcal{F}$ denotes the Fourier transformation. The projection tensor $\mathbf{P}(\mathbf{k})=\delta_{i j}-k_{i} k_{j} / k^{2}(i, j=1,2,3)$ projects $\mathcal{F}(\mathbf{u} \times \boldsymbol{\omega})$ onto the plane normal to the wavenumber vector $\mathbf{k}$ and eliminates the pressure gradient term in Eq. (1). The wavenumber components in Fourier space were defined as $k_{j}=n_{j}(2 \pi / L)$, where $n_{j}=-N / 2, \ldots,-1,0,1, \ldots, N / 2-1$ for $j=1,2,3$. The maximum wavenumber was approximately $N / 3$, and $N=512$ was set in the DNS. The spatial resolution was monitored by the value of $k_{\max } \eta$, where $\eta$ is the Kolmogorov length scale. The value of $k_{\max } \eta$ should be larger than 1.0 for the Kolmogorov scale of the flow to be well resolved, and it was always larger than 1.3 in our simulations. The fluid velocity in Fourier space was advanced in time using a secondorder Adams-Bashforth method for the nonlinear term and an exact integration for the linear viscous term. The time step was chosen to ensure that the Courant-Friedrichs-Lewy (CFL) number was 0.5 or less for numerical stability and accuracy. $\hat{\mathbf{f}}(\mathbf{k}, t)$ is the large-scale forcing term in the Fourier space. There are usually two types of large-scale forcing methods, that is, the deterministic method and stochastic forcing method. Generally, the type of large-scale forcing may affect the statistics of particle motions. This effect can be attributed to the changing Taylor microscale Reynolds number and turbulent structures in the flow under different forcing methods. ${ }^{51,52}$ The parameters in different methods should be properly set to avoid undesirable effects. For the stochastic forcing method, the forcing time scale $t_{f}$ is an important input parameter, and it is suggested to set $\Delta t<t_{f}<\tau_{K}$, where $\Delta t$ is the time step used to discretize the Navier-Stokes equations and $\tau_{K}$ is the Kolmogorov timescale. Under such a setting, statistics of both the flow and particle motion are not sensitive to the forcing time scale. ${ }^{51}$ While for the deterministic forcing method used in the present paper, the energy levels in the first two shells $(0.5<|\mathbf{k}|<1.5$ and $1.5<|\mathbf{k}|<2.5)$ are set to be $E(1)$ $=0.55544$ and $E(2)=0.159843$. The given values meet the $k^{-5 / 3}$ energy spectrum scaling in the inertial subrange. ${ }^{53}$ Chen et al. ${ }^{54}$ found that the type of large-scale forcing schemes with proper settings has little effect on the Lagrangian statistics of fluid particles in isotropic turbulence.

\section{B. Large-eddy simulation}

An LES of homogeneous and isotropic turbulence was performed at a much coarser grid resolution than the above DNS using the same pseudo-spectral method and large-scale forcing scheme. The governing equation in Fourier space for the LES is given by

$$
\left(\frac{\partial}{\partial t}+\left[v+v_{e}\left(k \mid k_{c}\right)\right] k^{2}\right) \hat{\overline{\mathbf{u}}}(\mathbf{k}, t)=\mathbf{P}(\mathbf{k}) \mathcal{F}(\overline{\mathbf{u}} \times \overline{\boldsymbol{\omega}})+\hat{\mathbf{f}}(\mathbf{k}, t),
$$

where $\overline{\mathbf{u}}$ and $\overline{\boldsymbol{\omega}}$ are the resolved velocity and vorticity in physical space, respectively. A spectral eddy-viscosity SGS model is used, ${ }^{55,56}$

$$
\begin{gathered}
v_{e}\left(k \mid k_{c}\right)=v_{e}^{+}\left(k \mid k_{c}\right) \sqrt{E\left(k_{c}\right) / k_{c}}, \\
v_{e}^{+}\left(k \mid k_{c}\right)=C_{k}^{-3 / 2}\left[0.441+15.2 \exp \left(-3.03 k_{c} / k\right)\right] .
\end{gathered}
$$

Here, $v_{e}\left(k \mid k_{c}\right)$ denotes the spectral eddy-viscosity and $k_{c}$ denotes the cutoff wavenumber in the LES. The quantity $E\left(k_{c}\right)$ is the value of the energy spectrum at the cutoff wavenumber, and $C_{k}=2.0$ was used in this work. The above SGS model is constructed based on the turbulent energy budget equations, which, in general, can predict the energy spectrum, especially at low wavenumbers. The hypothesis of a $k^{-5 / 3}$ energy spectrum up to $k_{c}$ was used to parameterize the SGS model. This hypothesis is believed to be better fulfilled in turbulent flows with increasing Reynolds numbers.

To evaluate the effects of different SGS models on the background turbulence and particle statistics, the classical Smagorinsky model is also used,

$$
\begin{gathered}
\tau_{i j}=-2\left(C_{S} \Delta\right)^{2}|\bar{S}| \bar{S}_{i j}+(1 / 3) \tau_{k k} \delta_{i j}, \\
|\bar{S}|=\left(2 \bar{S}_{i j} \bar{S}_{i j}\right)^{1 / 2},
\end{gathered}
$$

where $\bar{S}_{i j}=\frac{1}{2}\left(\frac{\partial \bar{u}_{i}}{\partial x_{j}}+\frac{\partial \bar{u}_{j}}{\partial x_{i}}\right)$ denotes the strain rate tensor and $C_{S}$ $=\frac{1}{\pi}\left(\frac{2}{3 C_{k}}\right)^{3 / 4} \approx 0.14$ denotes the Smagorinsky coefficient. ${ }^{5}$

The comparisons of energy spectra and statistics of fluid particle pairs and inertial particles obtained from the LES with spectral eddy-viscosity model and Smagorinsky model are shown in the Appendix, demonstrating that the type of different SGS models imposes marginal influences on the statistics of the background turbulence and the statistics of fluid and inertial particles studied in the present paper.

\section{Filtered direct numerical simulation}

The velocity field of the FDNS was obtained by applying a sharp spectral filter to the DNS velocity field, which removed all the Fourier modes of the velocity beyond the cutoff wavenumber $k_{c}$. The filtered velocity is given by

$$
\hat{\tilde{\mathbf{u}}}(\mathbf{k}, t)= \begin{cases}\hat{\mathbf{u}}(\mathbf{k}, t), & \text { if }|\mathbf{k}| \leq k_{c}, \\ 0, & \text { otherwise. }\end{cases}
$$

The Eulerian statistics of flow fields from the DNS, FDNS, and LES are listed in Table I. In isotropic turbulent flows with zero mean velocity, the Taylor Reynolds number $R e_{\lambda}$ is defined as

$$
R e_{\lambda}=u^{\prime} \lambda / v
$$

where $u^{\prime}=\sqrt{\left\langle u_{i} u_{i}\right\rangle / 3}$ is the root mean square (rms) of the turbulent fluctuating velocity and $\lambda=\left(15 v u^{\prime 2} / \varepsilon\right)^{1 / 2}$ is the Taylor microscale. Corresponding to the DNS $512^{3}$, the LES with $64^{3}$ grid resolution is performed with the closure of Eqs. (5) and (6). The FDNS with $k_{c}$ $=0.125 k_{\max }$ can be regarded as an ideal LES without any SGS model error. 
TABLE I. Eulerian statistics of flow fields from different cases.

\begin{tabular}{lccc}
\hline \hline Case & DNS & FDNS & LES \\
\hline Grid number $N^{3}$ & $512^{3}$ & $\ldots$ & $64^{3}$ \\
Maximum wavenumber $k_{\max }\left(k_{c}\right)$ & 170 & 21 & 21 \\
Grid length $d x$ & 0.012 & $\ldots$ & 0.098 \\
Kinematic viscosity $v$ & 0.0010 & 0.0010 & 0.0010 \\
Taylor Reynolds number $R e_{\lambda}$ & 205.51 & $\ldots$ & $\ldots$ \\
rms fluctuating velocity $u^{\prime}$ & 0.870 & 0.861 & 0.854 \\
Dissipation rate $\varepsilon$ & 0.204 & $\ldots$ & 0.199 \\
Spatial resolution $k_{\max } \eta$ & 1.432 & $\ldots$ & $\ldots$ \\
Kolmogorov length scale $\eta$ & 0.00835 & $\ldots$ & $\ldots$ \\
Kolmogorov time scale $\tau_{\eta}$ & 0.06976 & $\ldots$ & $\ldots$ \\
\hline \hline
\end{tabular}

\section{Inertial particle motion}

The dispersed phase is assumed to be a dilute suspension of inertial particles, which allows us to neglect the feedback effects of particle loading on the fluid flow. The diameter of particles $d_{p}$ is set to be smaller than the Kolmogorov length scale $\eta$, so we can simulate these particles as point particles. Then, the ratio of the particle density to the fluid density is assumed to be much larger than $1\left(\rho_{p} / \rho_{f}\right.$ $\gg 1$ ); here, we only consider the drag force; thus, the equations of motion for the particles can be written as

$$
\begin{gathered}
\frac{\mathrm{d} \mathbf{X}(t)}{\mathrm{d} t}=\mathbf{V}(t), \\
\frac{\mathrm{d} \mathbf{V}(t)}{\mathrm{d} t}=\frac{[\mathbf{u}(\mathbf{X}(t), t)-\mathbf{V}(t)] f}{\tau_{p}},
\end{gathered}
$$

where $\mathbf{X}(t)$ and $\mathbf{V}(t)$ are the particle position and velocity at time $t$, respectively; $\mathbf{u}(\mathbf{X}(t), t)$ is the fluid velocity seen by the particle, which can be calculated by a three-dimensional sixth-order Lagrangian interpolation scheme; $f\left(R e_{p}\right)=1+0.15 R e_{p}^{0.687}$ denotes the nonlinear drag coefficient, $R e_{p}=|\mathbf{u}-\mathbf{V}| d_{p} / v$ is the particle Reynolds number, $\tau_{p}=\rho_{p} d_{p}^{2} / 18 \rho_{f} v$ is the particle response time, and $d_{p}=0.4 \eta$. To characterize the inertia of particles, we define the Stokes number

$$
S t=\frac{\tau_{p}}{\tau_{\eta}} .
$$

Equations (11) and (12) are integrated in time using the fourthorder Adams-Moulton method and the fourth-order AdamsBashforth method, respectively.

We treat the particles as ghost particles and let the particle can overlap and then move independently. ${ }^{58}$ When the distance between two approaching particles becomes less than or equal to the diameter of the particle from a larger distance than the diameter, we can count the occurrence of a collision. In this work, we consider 20 different Stokes numbers, namely, St $=0.05,0.1,0.3,0.5,0.7,1.0,1.25$, $1.5,1.75,2.0,2.25,2.5,3.0,4.0,5.0,6.0,8.0,10.0,15.0$, and 20.0, and use 800000 particles for each St. These particles are introduced into the statistically stationary flow field at random positions and with the fluid velocity at those locations. After a sufficient time (4-6 eddy turnover times) to allow the particles to equilibrate with the flow, we calculate the statistics of particle clustering. To obtain statistically accurate results, the particle statistics are averaged over several eddy turnover times.

\section{KSAD HYBRID MODEL}

In this section, we present the KSAD model, which is employed in this work to predict the clustering of inertial particles in the LES. The KSAD model was proposed to simulate the contribution of missing small-scale turbulence on particle motion; ${ }^{32}$ this approach has been successfully applied to fluid particles to identify significant differences between LESs and DNSs in the literature. ${ }^{27}$

\section{A. Approximate deconvolution model}

Based on the velocity field in the LES, the unfiltered solution of the flow field at resolved scales can be approximated by applying an ADM,

$$
\hat{\mathbf{u}}^{*}=\hat{G}^{-1} \hat{\hat{\mathbf{u}}}
$$

where $\hat{\mathbf{u}}$ is the filtered velocity in the LES, $\hat{\mathbf{u}}^{*}$ is an approximation of the unfiltered velocity, and $\hat{G}^{-1}(k)$ is the inverse of $\hat{G}$, which can be approximated as 35,59

$$
\hat{G}^{-1}(k) \approx \sum_{n=0}^{N}(1-\hat{G}(k))^{n} .
$$

Here, we choose $N=5^{59}$ and a three-dimensional transfer function of Gaussian from

$$
\hat{G}(k)=\exp \left(-\frac{\left|\mathbf{k}^{2}\right| \Delta^{2}}{24}\right), \quad \Delta=\pi / k_{c} .
$$

The velocity $\hat{\mathbf{u}}^{*}$ can be calculated by the repeated filtering of $\hat{\overline{\mathbf{u}}}$,

$$
\hat{\mathbf{u}}^{*} \approx 6 \hat{\overline{\mathbf{u}}}-15 \hat{\overline{\overline{\mathbf{u}}}}+20 \hat{\overline{\overline{\mathbf{u}}}}-15 \hat{\overline{\overline{\mathbf{u}}}}+6 \hat{\overline{\overline{\overline{\mathbf{u}}}}}-\hat{\underline{\overline{\overline{\mathbf{u}}}}}
$$

The ADM is used to improve the energy spectrum, especially the dissipation spectrum, of the resolved part of the LES. The improved dissipation spectrum is further used to construct the KS based on Eq. (26) in Subsection III B.

\section{B. Kinematic simulation}

According to the velocity field of the LES+ADM approach, a KS model is used to compensate for the velocity fluctuations at subgrid scales. The standard form of the KS velocity field is ${ }^{42,60,61}$

$$
\mathbf{u}_{\mathrm{KS}}(\mathbf{x}, t)=\sum_{n=1}^{N_{k}} \sum_{m=1}^{M}\left\{\mathbf{a}_{n m} \cos \left(\mathbf{k}_{n m} \cdot \mathbf{x}\right)+\mathbf{b}_{n m} \sin \left(\mathbf{k}_{n m} \cdot \mathbf{x}\right)\right\},
$$

where $\mathbf{a}_{n m}$ and $\mathbf{b}_{n m}$ are the vector coefficients of the Fourier cosine and sine modes of the velocity, respectively; $\mathbf{k}_{n m}$ is the wavevector; and $\mathbf{x}$ is the coordinate vector. The indices " $\mathrm{nm}$ " are used in a spherical coordinate system, where " $n$ " denotes the magnitude of the wavenumber $k_{n}=\left|\mathbf{k}_{n m}\right|$ and " $m$ " is an index of randomly oriented wavevectors of magnitude $k_{n}$. To achieve the fastest possible convergence for fluid particle statistics, we used the geometric distribution of wavenumbers beyond the cutoff wavenumber, ${ }^{62}$

$$
k_{n}=k_{c}\left(\frac{k_{\max }}{k_{c}}\right)^{\frac{n-1}{N_{k}-1}},
$$




$$
\mathbf{k}_{n m}=k_{n}\left(\sin \theta_{n m} \cos \phi_{n m}, \sin \theta_{n m} \sin \phi_{n m}, \cos \theta_{n m}\right),
$$

where $n=1,2, \ldots, N_{k}$ and $k_{\max }$ is the largest wavenumber considered. The orientation angles $\theta_{n m}$ and $\phi_{n m}$ for the $M$ wavevectors associated with each wavenumber were chosen randomly from a uniform distribution over a spherical shell of radius $k_{n}$ :

$$
\cos \theta_{n m} \in[-1,1], \quad \phi_{n m} \in[0,2 \pi] .
$$

To ensure the incompressibility of the subgrid velocity $\mathbf{u}_{\mathrm{KS}}(\mathbf{x}, t)$, we define the coefficients $\mathbf{a}_{n m}$ and $\mathbf{b}_{n m}$ as

$$
\mathbf{a}_{n m}=\mathbf{A}_{n m} \times \hat{\mathbf{k}}_{n m}, \quad \mathbf{b}_{n m}=\mathbf{B}_{n m} \times \hat{\mathbf{k}}_{n m},
$$

where $\hat{\mathbf{k}}_{n m}=\mathbf{k}_{n m} / k_{n}$ is a unit vector aligned with the wavevector $\mathbf{k}_{n m}$. $\mathbf{A}_{n m}$ and $\mathbf{B}_{n m}$ are random vectors with independent and normally distributed components, each with a mean of zero and a variance of $\sigma_{n}^{2}$,

$$
\sigma_{n}^{2}=\frac{1}{M} E\left(k_{n}\right) \Delta k_{n}
$$

where $\Delta k_{n}=\left(k_{n+1}-k_{n-1}\right) / 2$ for $n \in\left[2, N_{k}-1\right], \Delta k_{1}=\left(k_{2}-k_{1}\right) / 2$, and $\Delta k_{N_{k}}=\left(k_{N_{k}}-k_{N_{k}-1}\right) / 2 . E\left(k_{n}\right)$ denotes the subgrid energy spectrum at $k_{n}$, which can be approximated by the DNS spectrum or a known model spectrum,

$$
\begin{gathered}
E(k)=C_{k} \varepsilon^{2 / 3} k^{-5 / 3} f_{\eta}(k \eta), \\
f_{\eta}(k \eta)=\exp \left\{-\beta\left\{\left[(k \eta)^{4}+c_{\eta}^{4}\right]^{1 / 4}-c_{\eta}\right\}\right\},
\end{gathered}
$$

where the dissipation rate is calculated from the LES+ADM approach and coupling between the ADM and KS is achieved,

$$
\varepsilon=\int_{0}^{k_{\max }} 2\left(v+v_{e}\right) k^{2} E(k) \mathrm{d} k,
$$

where the Kolmogorov length scale $\eta=\left(v^{3} / \varepsilon\right)^{1 / 4}$. Additionally, $C_{k}=2.0, \beta=5.2$, and $c_{\eta} \approx 0.15$.

After obtaining the incompressible subgrid velocity $\mathbf{u}_{\mathrm{KS}}(\mathbf{x}, t)$, the modeled velocity field can be written as

$$
\mathbf{u}_{\mathrm{MODEL}}(\mathbf{x}, t)=\mathbf{u}_{\mathrm{LES} / \mathrm{FDNS}}(\mathbf{x}, t)+\mathbf{u}_{\mathrm{KS}}(\mathbf{x}, t),
$$

where $\mathbf{u}_{\mathrm{LES} / \mathrm{FDNS}}(\mathbf{x}, t)$ is the velocity field from the LES+ADM approach or FDNS.

\section{RESULTS AND DISCUSSION}

\section{A. The KSAD model predictions of the RDF and RRV}

The RDF and RRV are the two key statistical inputs to the collision kernel for simulating finite-inertia particles suspended in turbulent flows; ${ }^{17,19}$ this topic will be systematically investigated using the KSAD model in this work. The RDF, which measures the level of particle clustering, can be computed from a field of $\mathrm{M}$ particles by binning the particles according to their separation distance as follows:

$$
g(r)=\frac{Q_{p, r} / \Delta V_{r}}{Q_{p} / V},
$$

where $Q_{p, r}$ is the average number of particles found in an elemental shell volume $\Delta V_{r}$ at a distance $r=|\mathbf{r}|$ from a reference particle, $V$ is the total volume, and $Q_{p}=N_{p}\left(N_{p}-1\right) / 2$ is the total number of particle pairs in the flow. The RRV is defined as

$$
w_{r}(\mathbf{r})=\left[\mathbf{V}_{2}(\mathbf{x}+\mathbf{r})-\mathbf{V}_{1}(\mathbf{x})\right] \cdot \frac{\mathbf{r}}{|\mathbf{r}|},
$$

where $\mathbf{V}_{1}(\mathbf{x})$ and $\mathbf{V}_{2}(\mathbf{x}+\mathbf{r})$ are the velocities of two particles located at $\mathbf{x}$ and $\mathbf{x}+\mathbf{r}$, respectively.

For a monodispersed particulate system, the average collision frequency is given by ${ }^{18}$

$$
\begin{gathered}
N_{c}=\frac{n_{0}^{2}}{2} K\left(d_{p}\right), \\
K\left(d_{p}\right)=2 \pi d_{p}^{2} g\left(d_{p}\right)\left\langle\left|w_{r}\right|\right\rangle,
\end{gathered}
$$

where $n_{0} \equiv N_{p} / V$ denotes the particle number density, $d_{p}$ is the particle diameter, and $K\left(d_{p}\right)$ is the collision kernel.

To assess the predictive ability of the KSAD model based on the statistics of inertial particle clustering in the LES, the RDF and RRV at different $S t$ from the LES+KSAD are compared with those from the DNS and LES.

Figures 1 and 2 show the RDF variations with $r / \eta$ from the DNS, LES, and LES+KSAD for $S t \leq 3.0$ and $S t>3.0$, respectively. Compared with that in the DNS, the lack of small-scale turbulence in the LES causes an underestimation of RDF at low St and an overestimation of RDF at high $S t$, with a crossover at approximately $S t$ $=1.5$. At low $S t$, the RDF in the LES is underestimated because particle clustering is primarily driven by the centrifugal forcing of particles out of the vortical regions and into the straining regions of the flow, and this centrifugal effect is strong at the small scales of turbulence. The absence of small-scale coherent turbulence for particles at low $S t$ leads to the underestimation of the RDF. For high $S t$, the particle response times are larger than the Kolmogorov time scale, and the small-scale turbulence acts as a random force and contributes to a comparatively low level of clustering. The lack of random smallscale turbulence leads to the overestimation of the RDF of particles at high $S t$. The crossover of the RDF at approximately $S t=1.5$ is caused by the competition between the centrifugal effect and random effect of small-scale turbulence on the particles.

In Figs. 1 and 2, we can observe the effect of the KSAD model on the variations in RDF for different $S t$. The KSAD model only partially reduces the error between the LES and DNS for $S t<2.0$. For $S t$ $\geq 2.0$, the results of the LES with KSAD model coincide well with the DNS results because the KSAD model recovers the turbulent kinetic energy at both the resolved and subgird scales in the LES (refer to Figs. 2 and 3 in our previous work ${ }^{32}$ ), thus enhancing the random motion of particles.

To intuitively show the effect of the KSAD model on particle clustering at different $S t$, we compare the particle distribution in a $2 \pi \times 2 \pi \times 10 \eta$ slice of the flow domain based on the DNS, LES, and LES+KSAD, as shown in Fig. 3. When $S t=0.5$, the lack of smallscale turbulence decreases the RDF, primarily at small separation levels, as shown in Fig. 1(c), and the particle distribution at large separation levels in the LES [Fig. 3(c)] is similar to that for the DNS [Fig. 3(a)]. When $S t=6.0$, the lack of small-scale turbulence reduces the random motion of all particles and increases the RDF at different 

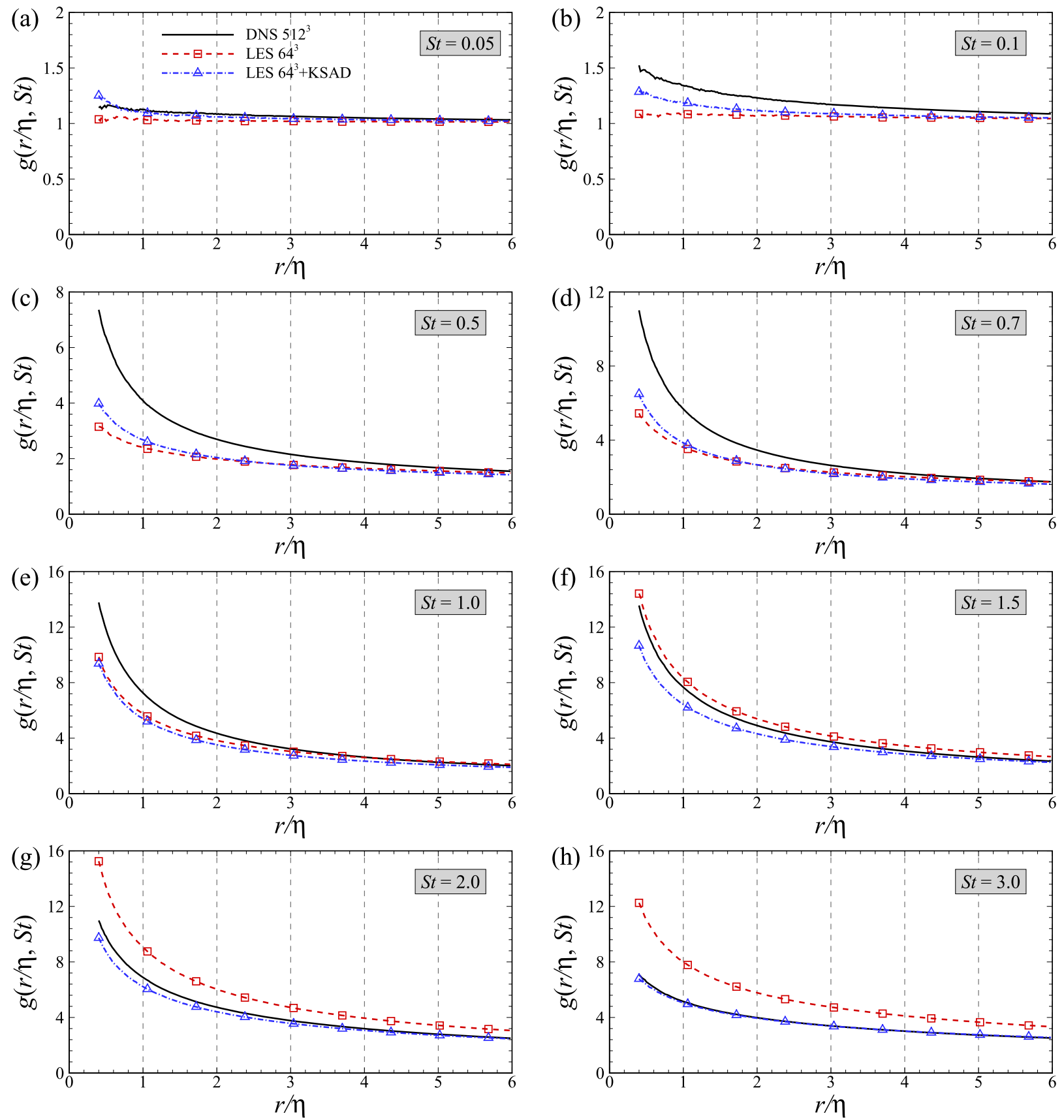

FIG. 1. Comparison of RDF variations with $r / \eta$ from the DNS, LES, and LES+KSAD for (a) $S t=0.05$, (b) $S t=0.1$, (c) $S t=0.5$, (d) $S t=0.7$, (e) $S t=1.0$, (f) $S t=1.5$, (g) $S t$ $=2.0$, and $(h) S t=3.0$.

separation levels, as shown in Fig. 2(b), resulting in enhanced clustering in the LES [Fig. 3(d)]. It can be observed that the KSAD model has a minor effect on the particle distribution at $S t=0.5$ but significantly reduces particle clustering at $S t=6.0$, as shown in Fig. 3(f), and thus improves the prediction.
Let us now consider the performance of the KSAD model based on the averaged RRV $\left\langle\left|w_{r}\right|\right\rangle$. Figures 4 and 5 show comparisons of the RRVs from the DNS, LES, and LES+KSAD for different St. The LES consistently underestimates the RRV for all $S t$, as opposed to the results for the RDF, compared with the DNS results due to the 

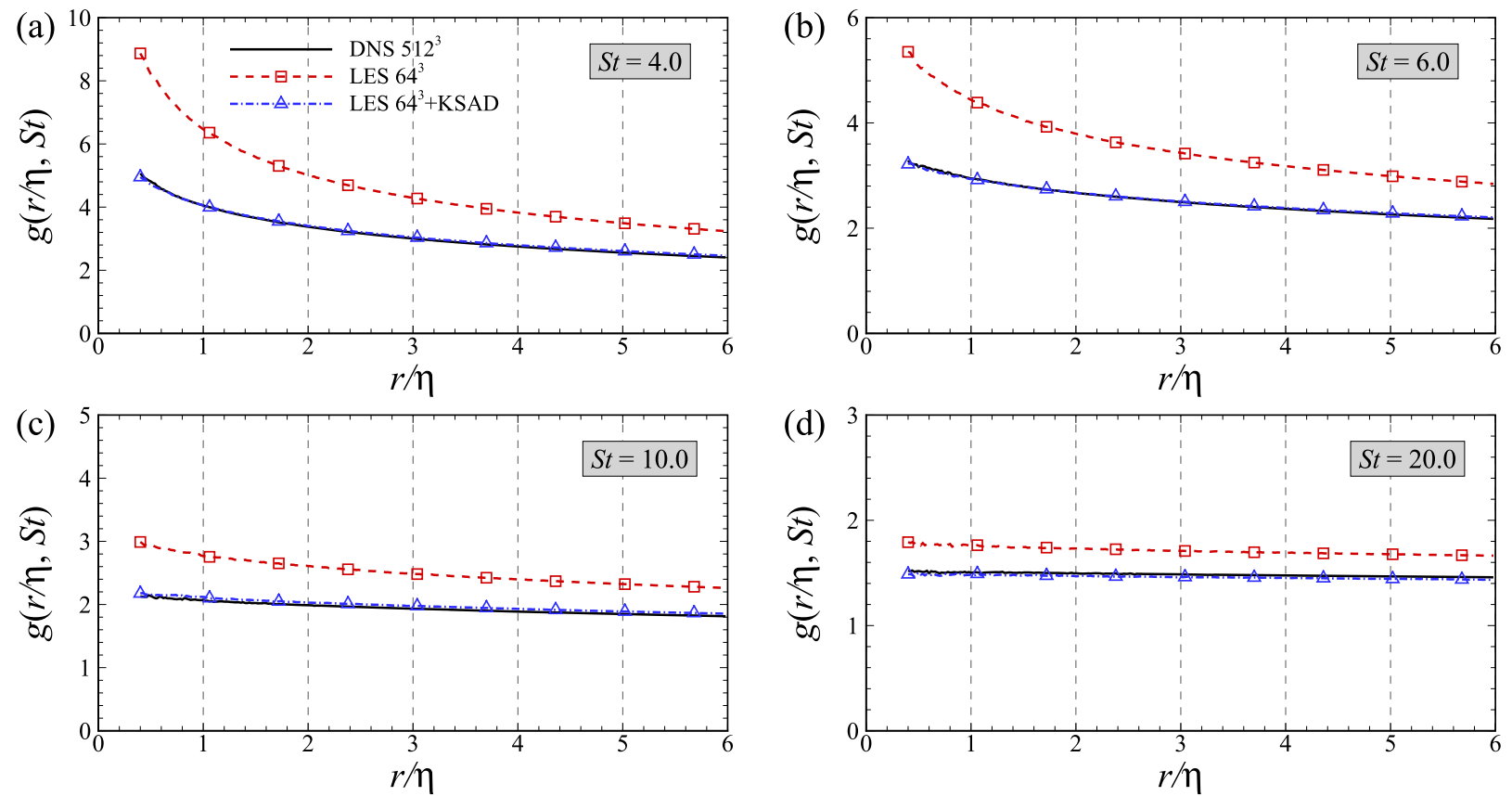

FIG. 2. Comparison of RDF variations with $r / \eta$ from the DNS, LES, and LES+KSAD for (a) $S t=4.0$, (b) $S t=6.0$, (c) $S t=10.0$, and (d) $S t=20.0$.

increase in coherence or improved correlation of velocity in the flow field obtained in the LES. ${ }^{23}$ The KSAD model quantitatively reduces the RRV error between the LES and DNS for both low and high St.

\section{B. The mechanisms of particle clustering}

To illustrate why the KSAD model cannot accurately predict the RDF at low $S t$, we compare the statistical properties of the flow fields from the DNS, FDNS, FDNS+KS, LES, and LES+KSAD and study the mechanisms of particle clustering. The FDNS with $k_{c}$ $=0.125 k_{\max }$ can be regarded as an a priori LES, and we choose the DNS spectrum as the input subgrid energy spectrum into the KS.

Figure 6 shows the probability density function (PDF) of dimensionless velocity gradient $\frac{\partial u / \partial x}{\sigma_{\partial u / \partial x}}$ and the value of $Q$ in the flow fields of the DNS, LES+KSAD, and FDNS+KS. $\sigma_{\partial u / \partial x}$ is the standard deviation of $\partial u / \partial x$, and $Q$ denotes the second invariant of the velocity gradient tensor $\nabla \mathbf{u},{ }^{63}$ defined as

$$
Q=\frac{1}{2}\left(\left\|\boldsymbol{\Omega}^{2}\right\|-\left\|\mathbf{S}^{2}\right\|\right)=-\frac{1}{2} \frac{\partial u_{i}}{\partial x_{j}} \frac{\partial u_{j}}{\partial x_{i}},
$$

where $\Omega_{i j}=\frac{1}{2}\left(\frac{\partial u_{i}}{\partial x_{j}}-\frac{\partial u_{j}}{\partial x_{i}}\right)$ denotes the vorticity tensor and $S_{i j}$ $=\frac{1}{2}\left(\frac{\partial u_{i}}{\partial x_{j}}+\frac{\partial u_{j}}{\partial x_{i}}\right)$ denotes the strain rate tensor. When $\tau_{p}$ is small, the particle velocity can be approximated as $\mathbf{V} \approx \mathbf{u}-\tau_{p} \mathbf{a}$, and this relation can be used to obtain the Eulerian particle velocity field as a function of the fluid velocity $\mathbf{u}$ and fluid acceleration $\mathbf{a}$ $=\mathrm{d} \mathbf{u} / \mathrm{d} t$. Then, the divergence of the particle velocity field can be approximated as ${ }^{64,65}$

$$
\nabla \cdot \mathbf{V} \approx-\tau_{p} \nabla \cdot \mathbf{a}=2 \tau_{p} Q
$$

According to Eq. (32), $Q$ is a measure of the balance between the vorticity and shear strain. At low $S t$, the particle velocity field will contract $(\nabla \cdot \mathbf{V}<0)$ in flow regions of low vorticity and high strain rate with negative $Q$ values. ${ }^{7}$ Positive $Q$ corresponds to vortex tube structures with high vorticity where the centrifugal force of eddies tends to drive the particles to the regions with negative $Q$.

In Fig. 6(a), the PDF of the dimensionless velocity gradient from the DNS has a negative skewness, and it is obviously larger in the middle and wider in the tails than the Gaussian distribution. Due to the missing small-scale turbulence, the PDFs from the FDNS and LES are much closer to the Gaussian distribution than that from the DNS. After generating the Gaussian turbulent-like flow field based on the KS, the PDFs from the FDNS+KS and LES+KSAD coincide well with the Gaussian distribution. In Fig. 6(b), the PDF of $Q$ from the DNS has much wider tails than that from the FDNS, demonstrating that large values of strain or vorticity are more likely in the flow field of the DNS. The PDFs of $Q$ from the FDNS+KS and LES+KSAD are wider than those from the FDNS and LES, but it cannot be recovered to the PDF of $Q$ from the DNS. According to Eq. (33), particle clustering at low $S t$ is closely related to $Q$, so that the difference in the PDFs of $Q$ between the FDNS+KS, LES+KSAD, and DNS will be reflected in the RDF.

To further illustrate the effect of $Q$ on inertial particle clustering in different flow fields, we track the inertial particles and calculate the value of $Q$ sampled along different particle paths. ${ }^{66}$ Figure 7 shows the PDF of dimensionless $Q / \sigma_{Q}$ sampled on the particle paths for fluid particles and inertial particles at different $S t$ from the DNS, FDNS, FDNS+KS, LES, and LES+KSAD, where $\sigma_{Q}$ is the standard deviation of $Q$. The PDF from the DNS first deflects to the left with increasing St starting from the curve of the fluid particle, which 

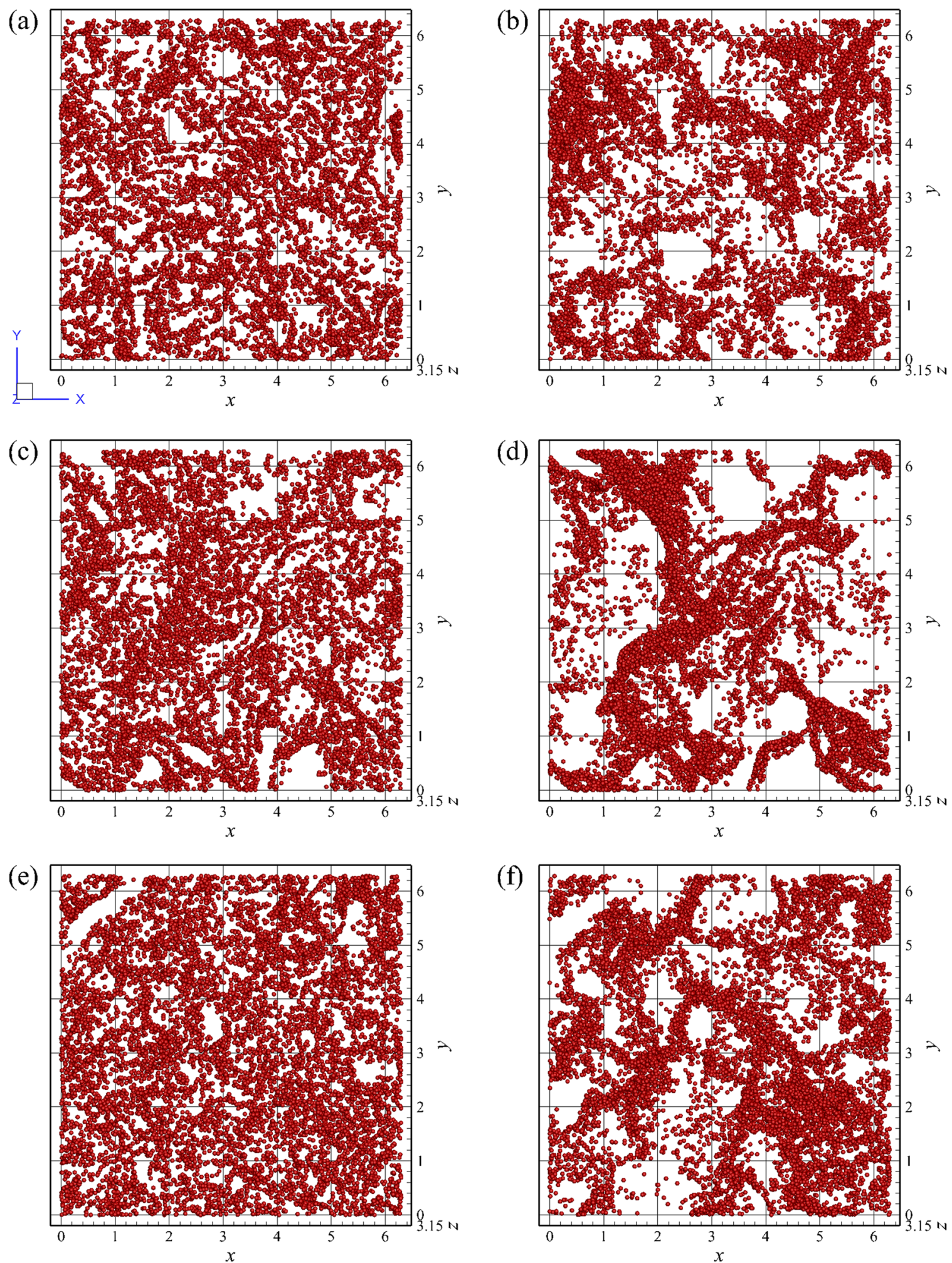

FIG. 3. Particle distribution for $S t=0.5[(\mathrm{a})$, (c), and (e)] and $S t=6.0[(\mathrm{~b})$, (d), and (f)] superimposed on top of a $2 \pi \times 2 \pi \times 10 \eta$ slice $(-5 \eta \leq z-L / 2 \leq 5 \eta)$ of the flow domain for $[(a)$ and $(b)]$ the DNS $512^{3}$, [(c) and (d)] the LES $64^{3}$, and [(e) and (f)] the LES $64^{3}+$ KSAD. 

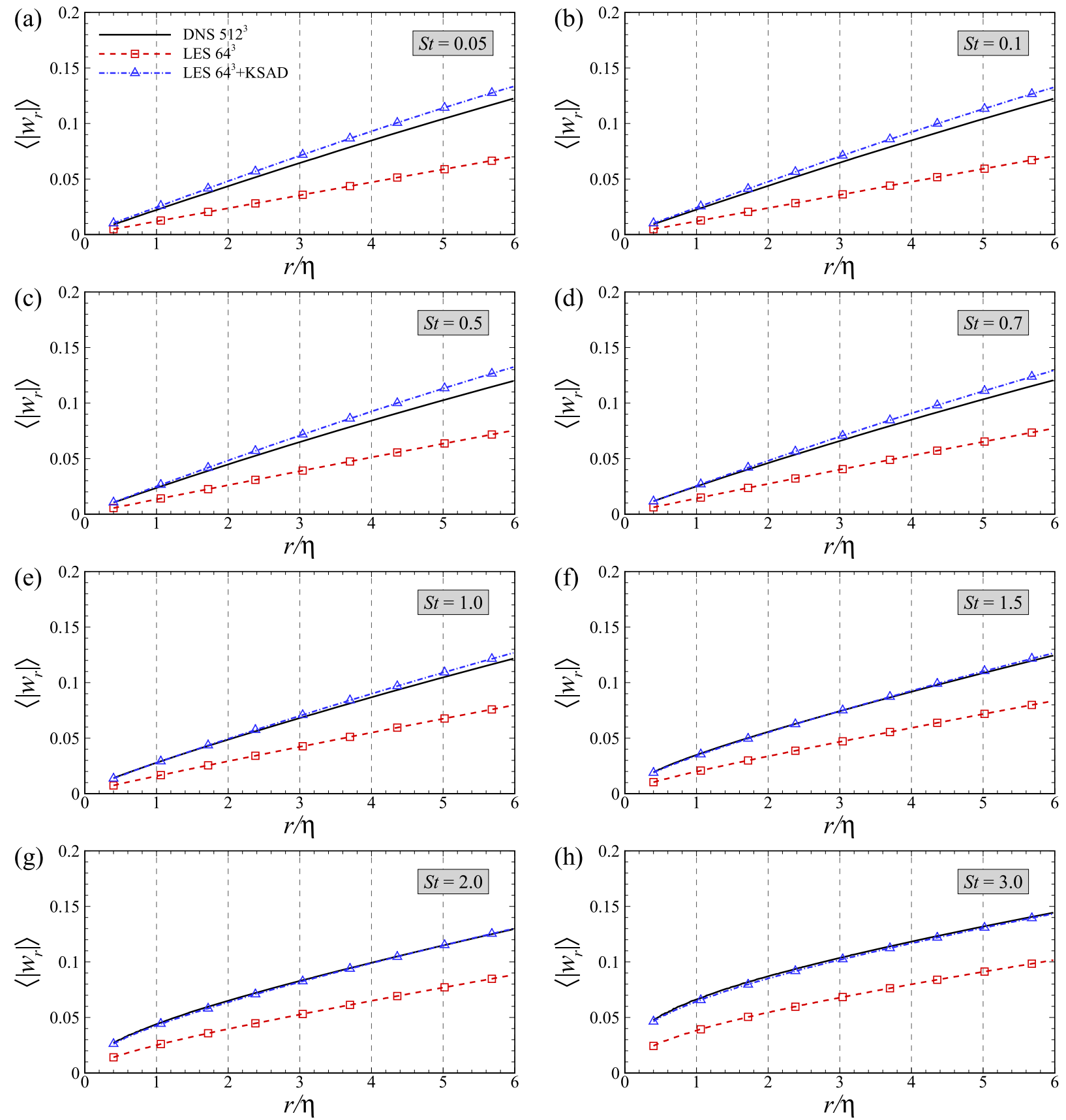

FIG. 4. Comparison of RRV variations with $r / \eta$ from the DNS, LES, and LES+KSAD for (a) $S t=0.05$, (b) $S t=0.1$, (c) $S t=0.5$, (d) $S t=0.7$, (e) $S t=1.0$, (f) $S t=1.5$, (g) $S t$ $=2.0$, and $(h) S t=3.0$.

means that the particles are most likely to appear in flow regions with negative $Q$ values. Then, the PDF deflects to the right after the critical value of $S t=0.3$, as shown in Fig. 7(a). At high St, for example, $S t=30.0$, the particles have response times much larger than the Kolmogorov time scale and they are almost unaffected by the small-scale turbulence, and the spatial distribution of inertial particles tends to become more and more uniform in the turbulent field. The effects due to preferential concentration and the consequently biased sampling of the velocity gradient tensor along particle trajectories are weakened, resulting in the PDF of $Q$ along particles at high 

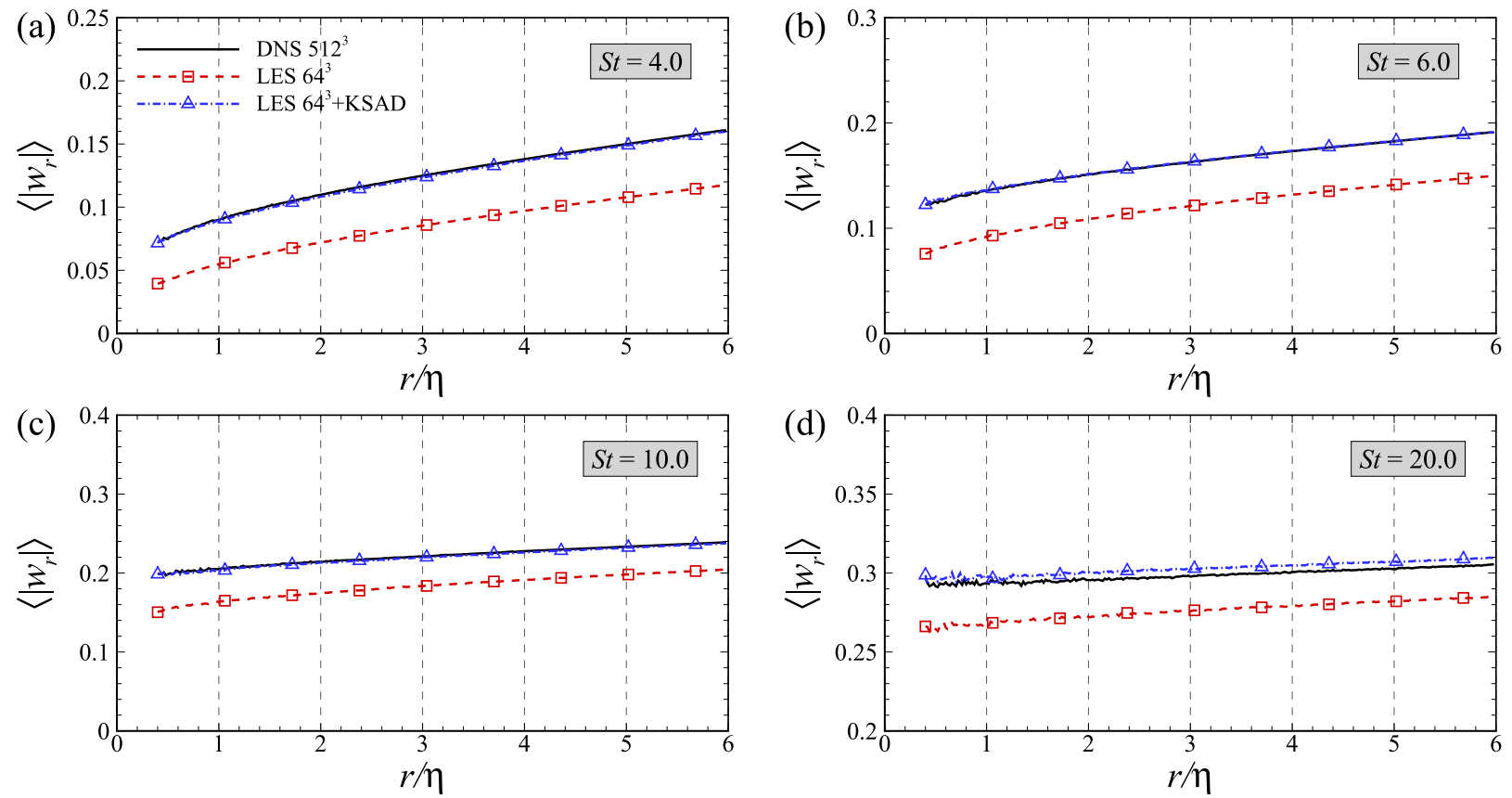

FIG. 5. Comparison of RRV variations with $r / \eta$ from the DNS, LES, and LES+KSAD for (a) $S t=4.0$, (b) $S t=6.0$, (c) $S t=10.0$, and (d) $S t=20.0$.

St tending to be consistent with the PDF curve of fluid particles. The absence of small-scale turbulence weakens the non-Gaussian nature of the system, which also reduces the differences in the PDFs at different $S t$ for the FDNS and LES. For the Gaussian turbulence in the FDNS+KS or LES+KSAD, the PDFs of $Q$ sampled on the inertial particle paths for all $S t$ are consistent with the curves of the fluid particles.

To quantitatively measure the asymmetry of the PDF of $Q$, we introduce the skewness of the PDF for $Q$ sampled on the particle paths; this variable is defined as

$$
S_{Q}=\frac{\left\langle(Q-\langle Q\rangle)^{3}\right\rangle}{\left\langle(Q-\langle Q\rangle)^{2}\right\rangle^{3 / 2}} .
$$

Figure 8 compares the variations in $S_{Q}$ and $S t \cdot S_{Q}$ with $S t$ from different simulations. In Fig. 8(a), the value of $S_{Q}$ in the DNS is mostly negative for $S t<1.0$, which suggests that the inertial particles are attracted to regions of negative $Q$ due to small-scale turbulence.
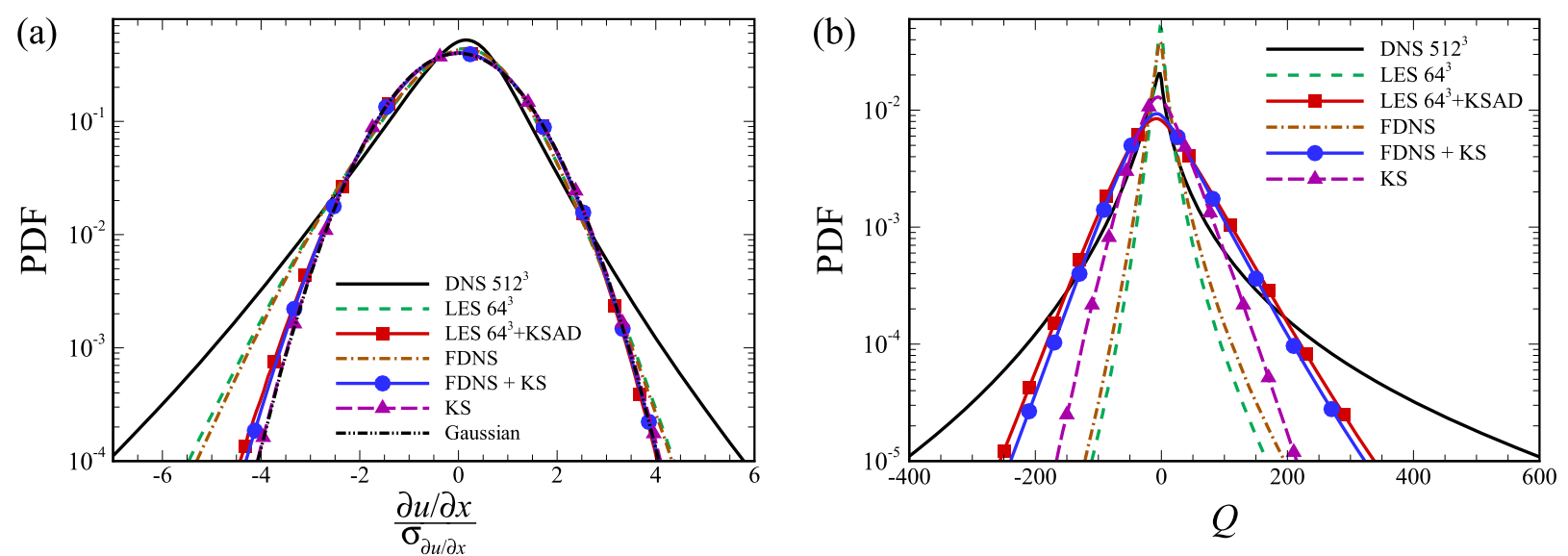

FIG. 6. PDFs of (a) $\frac{\partial u / \partial x}{\sigma_{\partial u / \partial x}}$ and (b) Q in the flow fields of the DNS, LES+KSAD, and FDNS+KS. 

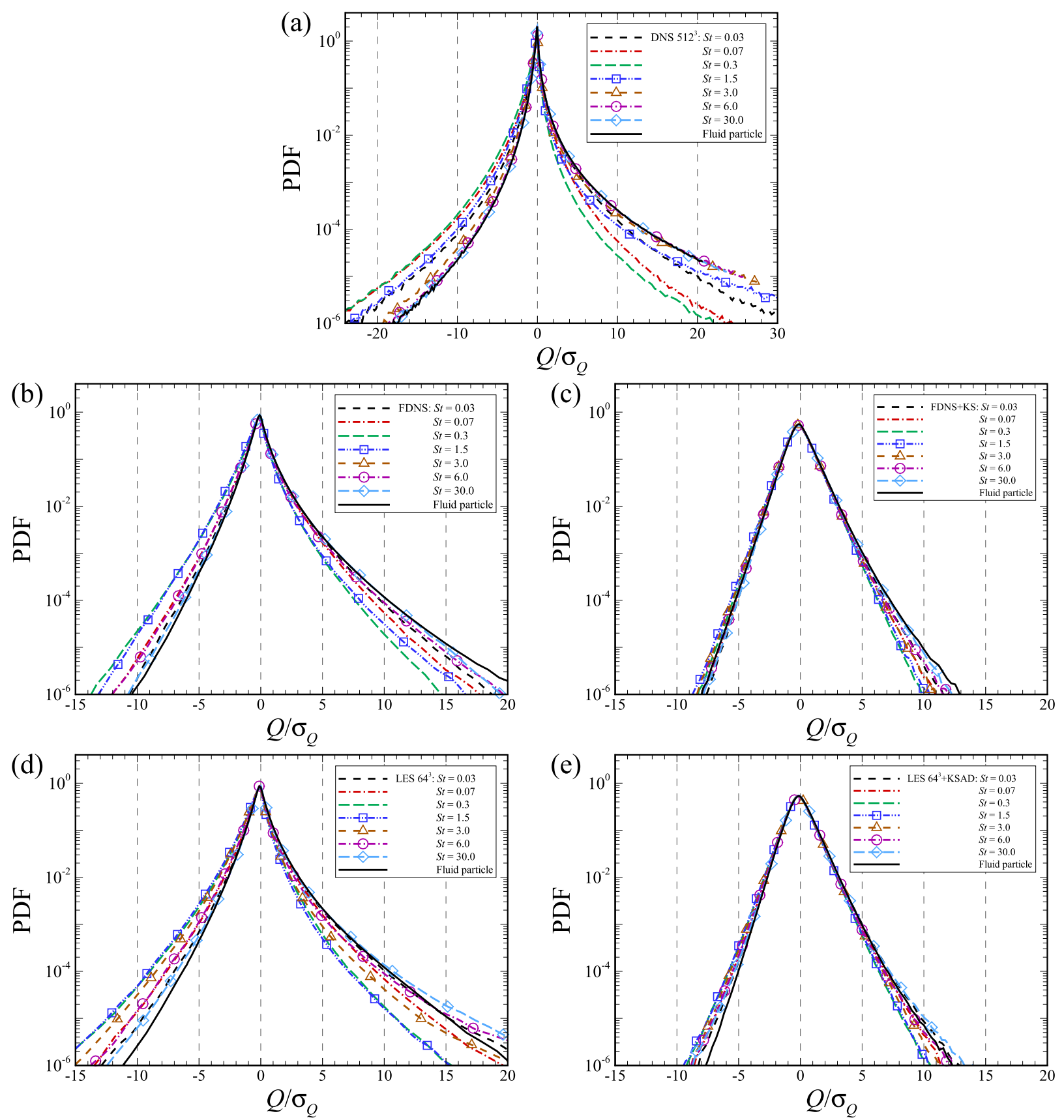

FIG. 7. PDF of dimensionless $Q / \sigma_{Q}$ sampled on the particle paths for fluid particles and inertial particles at different $S t$ from the (a) DNS, (b) FDNS, (c) FDNS+KS, (d) LES, and (e) LES+KSAD

The variations in $S_{Q}$ in the FDNS and LES are small compared to that found for the DNS. In the FDNS+KS or LES+KSAD, the variation in $S_{Q}$ almost plots as a horizontal line under Gaussian turbulence conditions. At low St, Eq. (33) directly represents the relation between particle clustering and the value of $S t \cdot S_{Q}$, as shown in Fig. 8(b). The differences in $S t \cdot S_{Q}$ among the DNS, LES, and LES+KSAD demonstrate that the KSAD model only partially improves the RDF prediction of the LES [see Figs. 1(b) and 1(c)] because KSAD is unable to reconstruct the intermittency of subgrid turbulence. When $S t \in[1.0$, 2.0 ], the KSAD model mainly compensates for the random effect of 
(a)

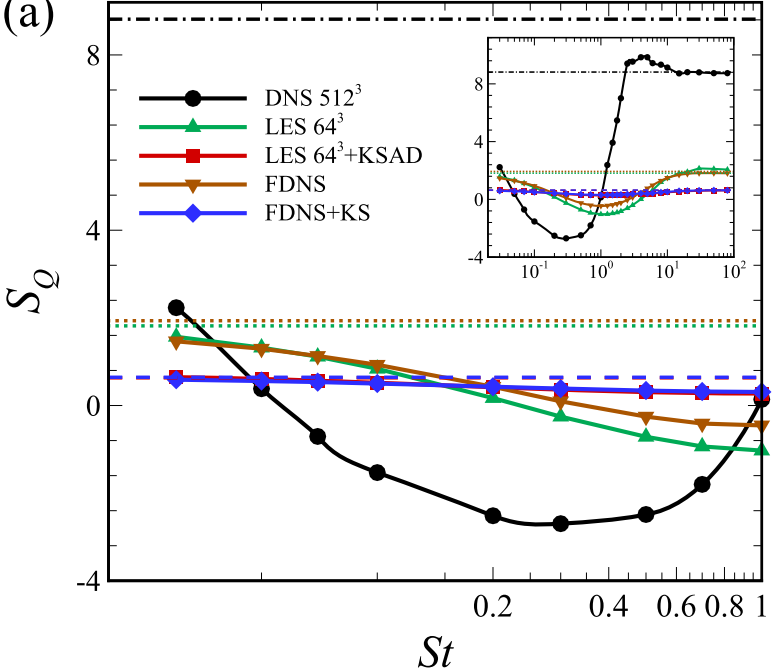

(b)

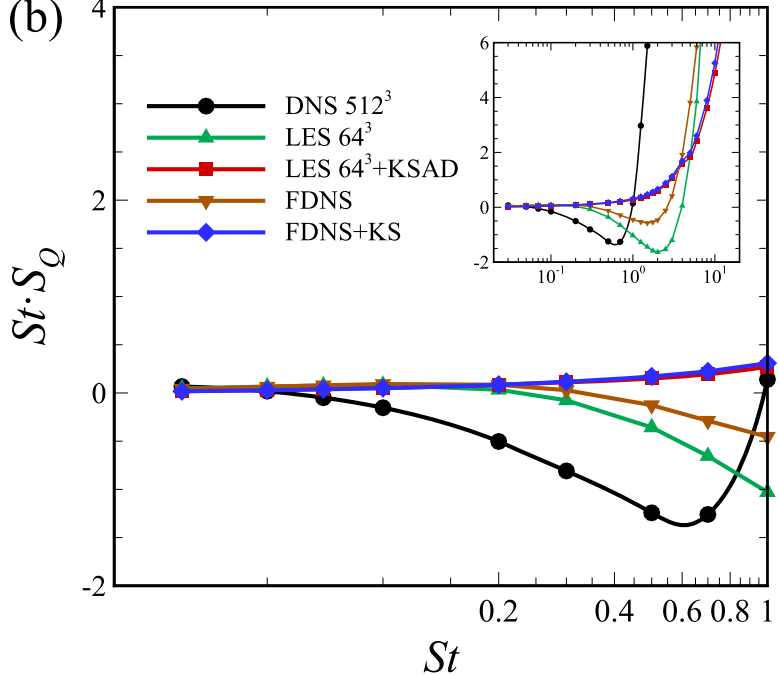

FIG. 8. (a) Skewness of the PDFs for $Q\left(S_{Q}\right)$ and (b) $S t$. $S_{Q}$ sampled on inertial particle paths at different $S t$ from the DNS and FDNS+KS. The symbols mark the numerical values, and the horizontal lines in (a) mark $S_{Q}$ for fluid particles from the DNS (upper, dashed-dotted line), FDNS and LES (middle, dotted lines), and FDNS+KS and LES+KSAD (lower, dashed lines).

small-scale turbulence but considers only a portion of the contribution of the centrifugal effect; therefore, the model cannot accurately predict the RDF in the vicinity of crossover between the LES and DNS. By further increasing St, the differences in the curves in Fig. 8(b) do not reflect particle clustering because Eq. (33) becomes invalid. In this stage, the LES+KSAD accurately predicts the DNS energy spectrum at both the resolved and subgrid scales; ${ }^{32}$ thus, the generated turbulence enhances the random motion of particles and reduces the RDF error between the LES and DNS (see Fig. 2).

\section{CONCLUSION}

In this paper, we test the performance of the KSAD model in predicting the RDF and RRV of inertial particles at different $S t$ in
LESs of isotropic turbulent flows by comparing the LES results with the DNS results. The LES without the KSAD model underestimates the RDF at low $S t$ and the RRV at all $S t$ but overestimates the RDF at high $S t$ due to the lack of subgrid-scale velocity fluctuations and the reductions in velocity fluctuations at resolved scales. However, the KSAD model is able to quantitatively predict the RRV for all $S t$ and the RDF for $S t \geq 2.0$, while the improvement in the predictions of $\mathrm{RDF}$ for $S t \leq 1.0$ is minor.

To explain the reason for the poor RDF predictions of the KSAD model at $S t<2.0$, we compare the Eulerian statistics and Lagrangian flow properties sampled on the particle paths from the DNS, FDNS, FDNS+KS, LES, and LES+KSAD and study the mechanisms of particle clustering. The PDFs of the velocity gradient and $Q$ from the DNS are obviously larger in the middle and wider in the
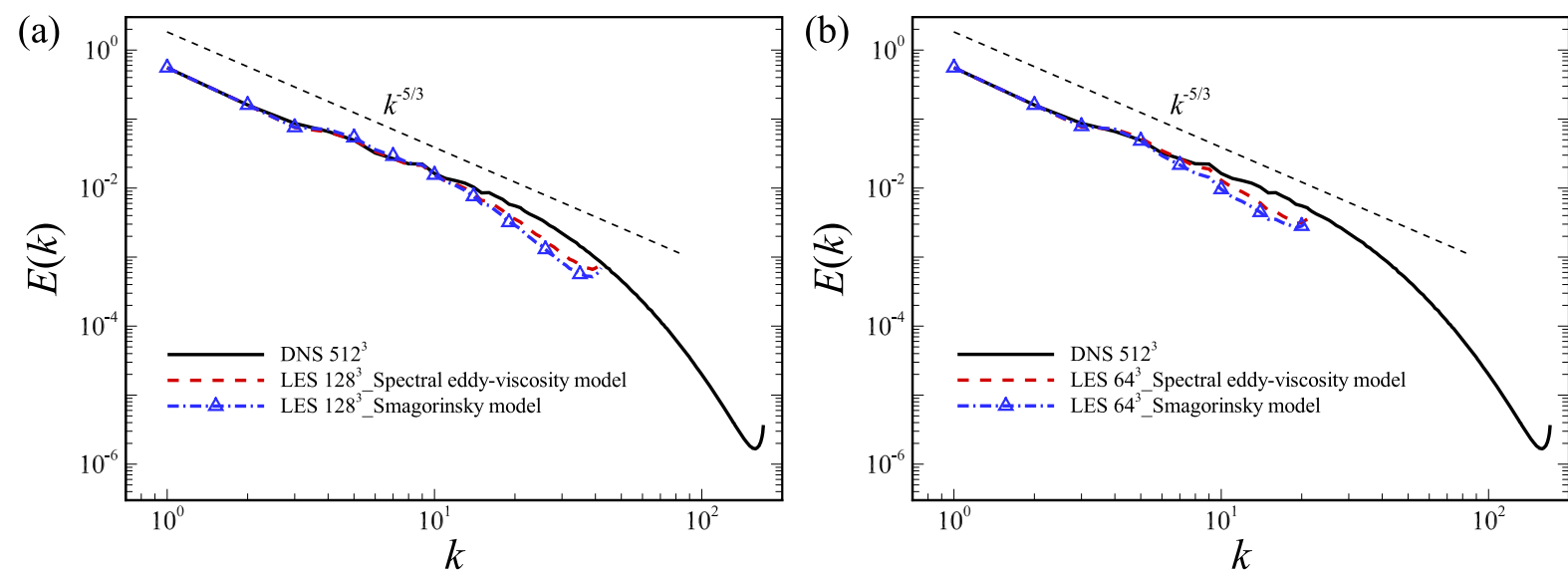

FIG. 9. Comparison of energy spectra for the DNS and LES with spectral eddy-viscosity model and Smagorinsky model. 
tails than those from the FDNS+KS. Furthermore, the comparisons of the PDFs of $Q$ sampled on the particle paths and the corresponding skewness demonstrate that the Gaussian turbulence generated by the KS model mainly compensates for the random effect and minimally considers the centrifugal effect of small-scale turbulence on particle clustering. Further efforts are needed to construct a more advanced model to recover the intermittency of the small-scale flow field missed in the LES of particle clustering in turbulent flows.

\section{ACKNOWLEDGMENTS}

This work was supported by the NSFC Basic Science Center Program for "Multiscale Problems in Nonlinear Mechanics" (Grant No. 11988102) and the NSFC Program (Grant No.11772337), the Science Challenge Program (Grant No. TZ2016001), the National Key Project (GJXM92579), the Strategic Priority Research Program, Chinese Academy of Sciences (CAS) (Grant No. XDB22040104),
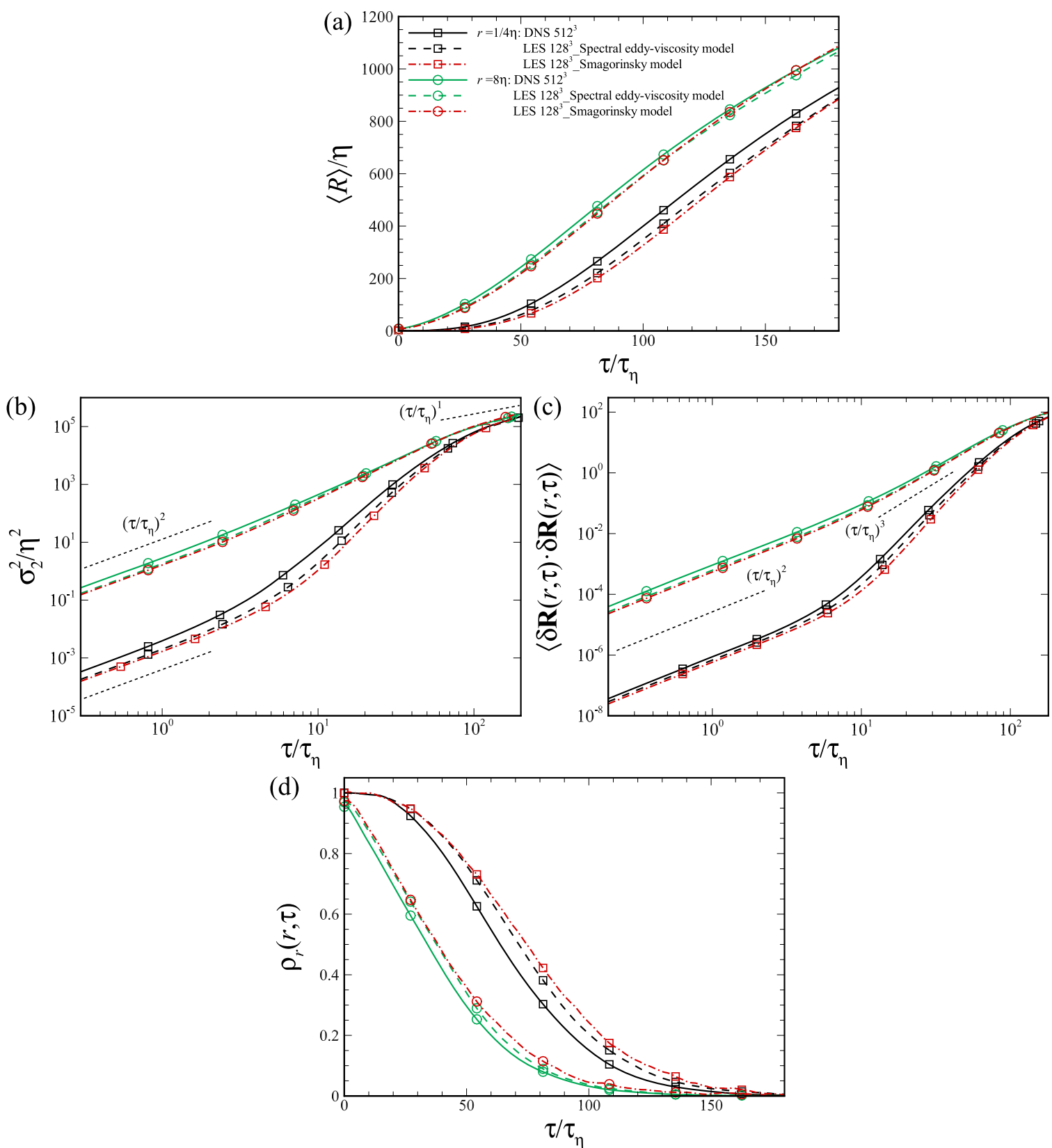

FIG. 10. Lagrangian statistics of fluid particle pairs in the DNS $512^{3}$ and LES $128^{3}$ with the spectral eddy-viscosity model and Smagorinsky model: (a) mean of the separation distance; (b) variance of the separation distance; (c) relative dispersion; and (d) one-time two-point Lagrangian velocity correlation function. 
the Key Research Program of Frontier Sciences, CAS (Grant No. QYZDJ-SSW-SYS002), and the CAS Center for Excellence in Complex System Mechanics.

\section{APPENDIX: THE EFFECT OF SGS MODELS ON FLOW AND PARTICLE STATISTICS}

To evaluate the effect of SGS models in the LES, we compare the energy spectra and statistics of fluid particle pairs and inertial particles in this appendix.

For a fluid particle pair, the separation distance and its variance are defined as

$$
\begin{gathered}
R\left(\mathbf{r}, t_{0} \mid \tau\right)=\sqrt{\mathbf{R}\left(\mathbf{r}, t_{0} \mid \tau\right) \cdot \mathbf{R}\left(\mathbf{r}, t_{0} \mid \tau\right),} \\
\mathbf{R}\left(\mathbf{r}, t_{0} \mid \tau\right)=\mathbf{X}\left(\mathbf{x}_{0}, t_{0} \mid t_{0}+\tau\right)-\mathbf{X}\left(\mathbf{x}_{0}+\mathbf{r}, t_{0} \mid t_{0}+\tau\right),
\end{gathered}
$$
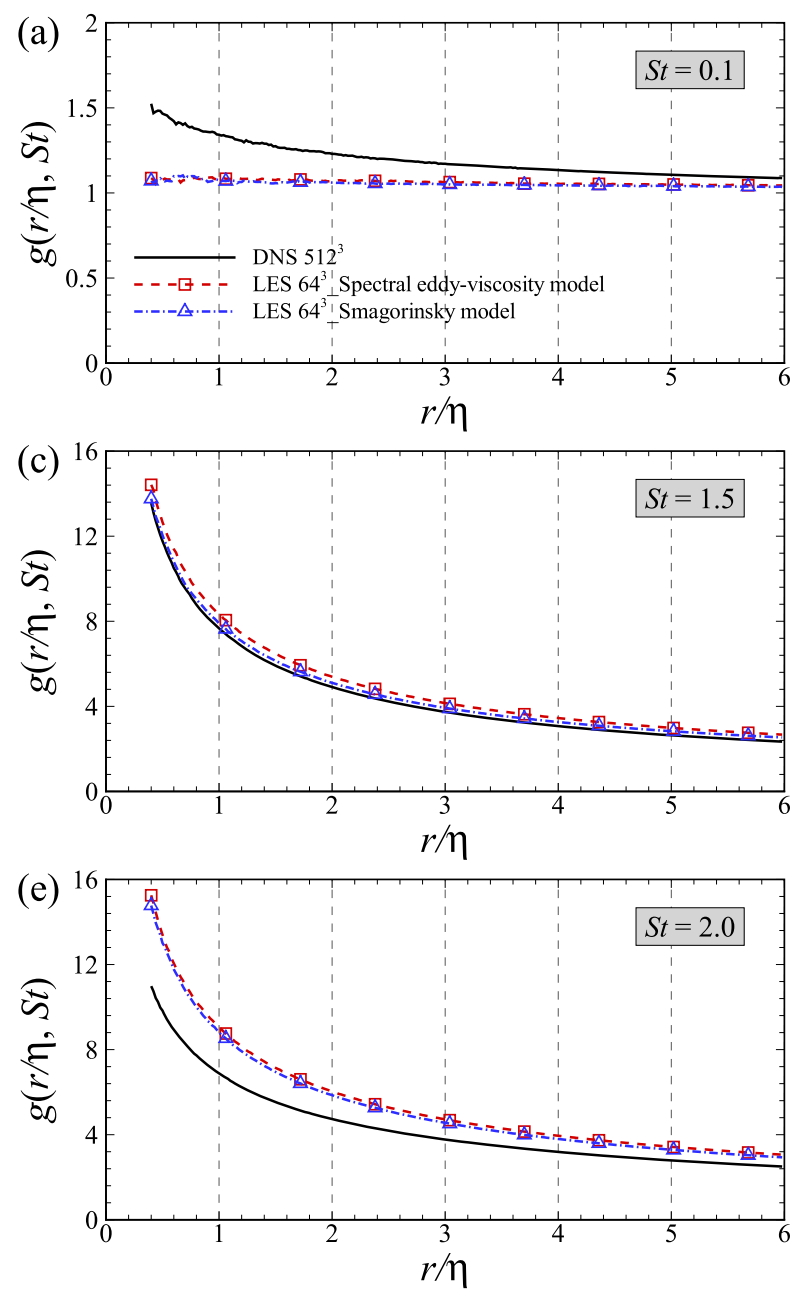

$$
\sigma_{2}^{2}(r, \tau)=\left\langle\mathbf{R}\left(\mathbf{r}, t_{0} \mid \tau\right) \cdot \mathbf{R}\left(\mathbf{r}, t_{0} \mid \tau\right)\right\rangle-\left\langle R\left(\mathbf{r}, t_{0} \mid \tau\right)\right\rangle^{2},
$$

where $\mathbf{R}\left(\mathbf{r}, t_{0} \mid \tau\right)$ denotes the separation vector of the particle pair, $\mathbf{r}$ is the initial separation vector, $r=|\mathbf{r}|$ is the initially prescribed separation distance, $\tau$ is the time lag, and \langle\rangle denotes the average over the particle pairs. The relative dispersion is defined as $\langle\delta \mathbf{R}(r, \tau) \cdot \delta \mathbf{R}(r, \tau)\rangle$, where $\delta \mathbf{R}(r, \tau)=\mathbf{R}\left(\mathbf{r}, t_{0} \mid \tau\right)-\mathbf{r}$ is the separation vector increment. The one-time two-point Lagrangian velocity correlation function is defined as

$$
\rho_{r}(r, \tau)=\frac{\left\langle\mathbf{V}\left(\mathbf{x}_{0}, t_{0} \mid t_{0}+\tau\right) \cdot \mathbf{V}\left(\mathbf{x}_{0}+\mathbf{r}, t_{0} \mid t_{0}+\tau\right)\right\rangle}{\left\langle\mathbf{V}\left(\mathbf{x}_{0}, t_{0}\right) \cdot \mathbf{V}\left(\mathbf{x}_{0}, t_{0}\right)\right\rangle},
$$

where $\mathbf{V}\left(\mathbf{x}_{0}, t_{0}\right)$ denotes the Lagrangian velocity of a fluid particle located at $\mathbf{x}_{0}$ at the initial time $t_{0}$ and $\mathbf{V}\left(\mathbf{x}_{0}, t_{0} \mid t_{0}+\tau\right)$ denotes the Lagrangian velocity of that fluid particle at time $t_{0}+\tau$.

Figure 9 compares the energy spectra for the DNS and LES with different SGS models. Compared to the spectral eddy-viscosity
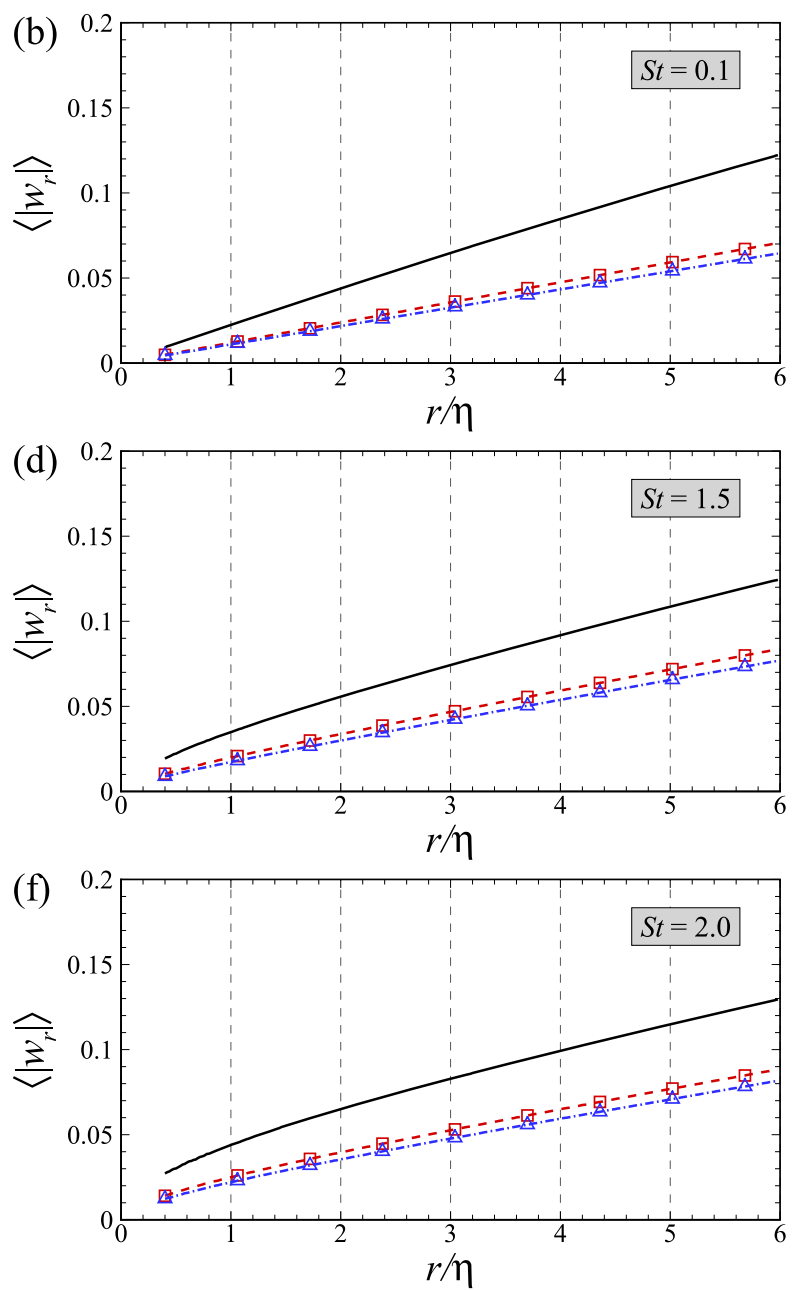

FIG. 11. Comparison of RDF and RRV variations with $r / \eta$ from the DNS $512^{3}$ and LES $64^{3}$ with the spectral eddy-viscosity model and Smagorinsky model at [(a) and (b)] St $=0.1,[(c)$ and $(d)] S t=1.5$, and $[(e)$ and $(f)] S t=2.0$. 
model, the Smagorinsky model slightly underestimates the energy spectrum near the cutoff wavenumber due to the excessive dissipation. The excessive dissipation also reduces the velocity fluctuations at the resolved scales, which causes the slower separation of fluid particle pairs and more correlated flow fields. Thus, the LES with Smagorinsky model slightly underestimates the mean and variance of the separation distance and the relative dispersion and overestimates the one-time two-point Lagrangian velocity correlation function, shown in Fig. 10.

Figure 11 plots the comparison of RDF and RRV from the DNS $512^{3}$ and LES $64^{3}$ with the spectral eddy-viscosity model and Smagorinsky model. The statistics of inertial particles at various Stokes numbers from the LES with Smagorinsky model are basically consistent with those from the LES with spectral eddy-viscosity model.

Overall, the type of SGS models imposes marginal influences on the statistics of the background turbulence and the statistics of fluid and inertial particles studied in the present paper.

\section{DATA AVAILABILITY}

The data that support the findings of this study are available from the corresponding author upon reasonable request.

\section{REFERENCES}

${ }^{1}$ H. T. Bi, N. Ellis, I. A. Abba, and J. R. Grace, "A state-of-the-art review of gassolid turbulent fluidization,” Chem. Eng. Sci. 55, 4789-4825 (2000).

${ }^{2}$ S. Som and S. K. Aggarwal, "Effects of primary breakup modeling on spray and combustion characteristics of compression ignition engines," Combust. Flame 157, 1179-1193 (2010).

${ }^{3}$ B. J. Devenish, P. Bartello, J.-L. Brenguier, L. R. Collins, W. W. Grabowski, R. H. A. IJzermans, S. P. Malinowski, M. W. Reeks, J. C. Vassilicos, L.-P. Wang, and Z. Warhaft, "Droplet growth in warm turbulent clouds," Q. J. R. Meteorol. Soc. 138, 1401-1429 (2012).

${ }^{4}$ J. T. Steffens, D. K. Heist, S. G. Perry, and K. M. Zhang, "Modeling the effects of a solid barrier on pollutant dispersion under various atmospheric stability conditions," Atmos. Environ. 69, 76-85 (2013).

${ }^{5} \mathrm{~W}$. W. Grabowski and L.-P. Wang, "Growth of cloud droplets in a turbulent environment," Annu. Rev. Fluid Mech. 45, 293-324 (2013).

${ }^{6}$ K. D. Squires and J. K. Eaton, "Preferential concentration of particles by turbulence," Phys. Fluids A 3, 1169 (1991).

${ }^{7}$ J. K. Eaton and J. R. Fessler, "Preferential concentration of particles by turbulence," Int. J. Multiphase Flow 20, 169-209 (1994).

${ }^{8}$ L.-P. Wang and M. R. Maxey, "Settling velocity and concentration distribution of heavy particles in homogeneous isotropic turbulence," J. Fluid Mech. 256, 27-68 (1993).

${ }^{9}$ A. Aliseda, A. Cartellier, F. Hainaux, and J. C. Lasheras, "Effect of preferential concentration on the settling velocity of heavy particles in homogeneous isotropic turbulence," J. Fluid Mech. 468, 77-105 (2002).

${ }^{10}$ R. A. Shaw, W. C. Reade, L. R. Collins, and J. Verlinde, "Preferential concentration of cloud droplets by turbulence: Effects on the early evolution of cumulus cloud droplet spectra," J. Atmos. Sci. 55, 1965-1976 (1998).

${ }^{11}$ P. A. Vaillancourt and M. K. Yau, "Review of particle-turbulence interactions and consequences for cloud physics," Bull. Am. Meteorol. Soc. 81, 285-298 (2000).

${ }^{12}$ W. C. Reade and L. R. Collins, "A numerical study of the particle size distribution of an aerosol undergoing turbulent coagulation," J. Fluid Mech. 415, 45-64 (2000).

${ }^{13}$ G. Falkovich, A. Fouxon, and M. G. Stepanov, "Acceleration of rain initiation by cloud turbulence," Nature 419, 151-154 (2002).
${ }^{14}$ R. A. Shaw, "Particle-turbulence interactions in atmospheric clouds," Annu. Rev. Fluid Mech. 35, 183-227 (2003).

${ }^{15} \mathrm{~L}$. Bourouiba, "Turbulent gas clouds and respiratory pathogen emissions: Potential implications for reducing transmission of COVID-19," JAMA 323(18), 1837-1838 (2020).

${ }^{16}$ R. Mittal, R. Ni, and J.-H. Seo, “The flow physics of COVID-19," J. Fluid Mech. 894, F2 (2020).

${ }^{17}$ S. Sundaram and L. R. Collins, "Collision statistics in an isotropic particle-laden turbulent suspension. Part 1. Direct numerical simulations," J. Fluid Mech. 335, 75-109 (1997).

${ }^{18}$ L.-P. Wang, A. S. Wexler, and Y. Zhou, "Statistical mechanical description and modelling of turbulent collision of inertial particles," J. Fluid Mech. 415, 117-153 (2000).

${ }^{19}$ B. Ray and L. R. Collins, "Preferential concentration and relative velocity statistics of inertial particles in Navier-Stokes turbulence with and without filtering," J. Fluid Mech. 680, 488-510 (2011).

${ }^{20} \mathrm{P}$. Moin and K. Mahesh, "Direct numerical simulation: A tool in turbulence research," Annu. Rev. Fluid Mech. 30, 539-578 (1998).

${ }^{21}$ T. Ishihara, T. Gotoh, and Y. Kaneda, "Study of high-Reynolds number isotropic turbulence by direct numerical simulation,” Annu. Rev. Fluid Mech. 41, 165-180 (2009).

${ }^{22}$ V. Armenio, U. Piomelli, and V. Fiorotto, "Effect of the subgrid scales on particle motion," Phys. Fluids 11, 3030 (1999).

${ }^{23}$ G.-W. He, G. Jin, and Y. Yang, "Space-time correlations and dynamic coupling in turbulent flows," Annu. Rev. Fluid Mech. 49, 51-70 (2017).

${ }^{24}$ G.-W. He, R. Rubinstein, and L.-P. Wang, "Effects of subgrid-scale modeling on time correlations in large eddy simulation," Phys. Fluids 14, 2186 (2002).

${ }^{25}$ G.-W. He, M. Wang, and S. K. Lele, "On the computation of space-time correlations by large-eddy simulation,” Phys. Fluids 16, 3859 (2004).

${ }^{26}$ Y. Yang, G.-W. He, and L.-P. Wang, "Effects of subgrid-scale modeling on Lagrangian statistics in large-eddy simulation," J. Turbul. 9, N8 (2008).

${ }^{27} \mathrm{Z}$. Zhou, J. Chen, and G. Jin, "Prediction of Lagrangian dispersion of fluid particles in isotropic turbulent flows using large-eddy simulation method," Acta Mech. 228, 3203-3222 (2017).

${ }^{28}$ G.-W. He and J.-B. Zhang, "Elliptic model for space-time correlations in turbulent shear flows," Phys. Rev. E 73, 055303 (2006).

${ }^{29} \mathrm{X}$. Zhao and G.-W. He, "Space-time correlations of fluctuating velocities in turbulent shear flows," Phys. Rev. E 79, 046316 (2009).

${ }^{30} \mathrm{P}$. Fede and O. Simonin, "Numerical study of the subgrid fluid turbulence effects on the statistics of heavy colliding particles," Phys. Fluids 18, 045103 (2006).

${ }^{31}$ G. Jin, G.-W. He, and L.-P. Wang, "Large-eddy simulation of turbulent collision of heavy particles in isotropic turbulence," Phys. Fluids 22, 055106 (2010).

${ }^{32}$ Z. D. Zhou, S. Z. Wang, and G. D. Jin, "A structural subgrid-scale model for relative dispersion in large-eddy simulation of isotropic turbulent flows by coupling kinematic simulation with approximate deconvolution method," Phys. Fluids $\mathbf{3 0}$, 105110 (2018).

${ }^{33}$ J. G. M. Kuerten and A. W. Vreman, "Can turbophoresis be predicted by largeeddy simulation?,” Phys. Fluids 17, 011701 (2005).

${ }^{34}$ C. Marchioli, M. V. Salvetti, and A. Soldati, "Some issues concerning large-eddy simulation of inertial particle dispersion in turbulent bounded flows," Phys. Fluids 20, 040603 (2008).

${ }^{35}$ S. Stolz and N. A. Adams, "An approximate deconvolution procedure for largeeddy simulation," Phys. Fluids 11, 1699 (1999).

${ }^{36}$ B. Shotorban and F. Mashayek, "Modeling subgrid-scale effects on particles by approximate deconvolution," Phys. Fluids 17, 081701 (2005).

${ }^{37}$ J. G. M. Kuerten, "Subgrid modeling in particle-laden channel flow," Phys. Fluids 18, 025108 (2006).

${ }^{38}$ M. J. Cernick, S. W. Tullis, and M. F. Lightstone, "Particle subgrid scale modelling in large-eddy simulations of particle-laden turbulence," J. Turbul. 16(2), 101-135 (2014).

${ }^{39}$ B. Shotorban and F. Mashayek, "A stochastic model for particle motion in largeeddy simulation,” J. Turbul. 7, N18 (2006). 
${ }^{40} \mathrm{~J}$. Pozorski and S. V. Apte, "Filtered particle tracking in isotropic turbulence and stochastic modeling of subgrid-scale dispersion," Int. J. Multiphase Flow 35, 118-128 (2009).

${ }^{41}$ G. Jin and G.-W. He, "A nonlinear model for the subgrid timescale experienced by heavy particles in large eddy simulation of isotropic turbulence with a stochastic differential equation," New J. Phys. 15, 035011 (2013).

${ }^{42}$ B. Ray and L. R. Collins, "A subgrid model for clustering of high-inertia particles in large-eddy simulations of turbulence," J. Turbul. 15, 366-385 (2014).

${ }^{43}$ M. Bassenne, M. Esmaily, D. Livescu, P. Moin, and J. Urzay, "A dynamic spectrally enriched subgrid-scale model for preferential concentration in particleladen turbulence," Int. J. Multiphase Flow 116, 270-280 (2019).

${ }^{44}$ G. I. Park, M. Bassenne, J. Urzay, and P. Moin, "A simple dynamic subgrid-scale model for LES of particle-laden turbulence,” Phys. Rev. Fluids 2, 044301 (2017).

${ }^{45} \mathrm{R}$. Maulik and O. San, "A neural network approach for the blind deconvolution of turbulent flows," J. Fluid Mech. 831, 151-181 (2017).

${ }^{46}$ R. Maulik, O. San, A. Rasheed, and P. Vedula, "Data-driven deconvolution for large eddy simulations of Kraichnan turbulence," Phys. Fluids 30, 125109 (2018).

${ }^{47}$ C. Y. Xie, J. C. Wang, H. Li, M. P. Wan, and S. Y. Chen, "Artificial neural network mixed model for large eddy simulation of compressible isotropic turbulence," Phys. Fluids 31, 085112 (2019).

${ }^{48}$ Z. Zhou, G.-W. He, S. Wang, and G. Jin, "Subgrid-scale model for largeeddy simulation of isotropic turbulent flows using an artificial neural network," Comput. Fluids 195, 104319 (2019).

${ }^{49}$ Z. W. Deng, C. X. He, Y. Z. Liu, and K. C. Kim, "Super-resolution reconstruction of turbulent velocity fields using a generative adversarial network-based artificial intelligence framework," Phys. Fluids 31, 125111 (2019).

${ }^{50}$ B. Liu, J. Tang, H. Huang, and X.-Y. Lu, "Deep learning methods for superresolution reconstruction of turbulent flows," Phys. Fluids 32, 025105 (2020).

${ }^{51}$ B. Rosa, H. Parishani, O. Ayala, and L.-P. Wang, "Effects of forcing time scale on the simulated turbulent flows and turbulent collision statistics of inertial particles," Phys. Fluids 27, 015105 (2015).

${ }^{52}$ R. Dhariwal and S. L. Rani, "Effects of deterministic and stochastic forcing schemes on the relative motion of inertial particles in DNS of isotropic turbulence," Powder Technol. 339, 46-69 (2018).
${ }^{53}$ S. Chen and X. Shan, "High-resolution turbulent simulations using the Connection Machine-2," Comput. Phys. 6, 643 (1992).

${ }^{54}$ J.-C. Chen, G.-D. Jin, and J. Zhang, "Lagrangian statistics in isotropic turbulent flows with deterministic and stochastic forcing schemes," Acta Mech. Sin. 31, 2531 (2015).

${ }^{55}$ J.-P. Chollet and M. Lesieur, "Parameterization of small scales of threedimensional isotropic turbulence utilizing spectral closures," J. Atmos. Sci. 38, 2747-2757 (1981).

${ }^{56}$ J. P. Chollet, "Two-point closure used for a sub-grid scale model in large eddy simulations," in Turbulent Shear Flows 4, edited by L. J. S. Bradbury, F. Durst, B. E. Launder, F. W. Schmidt, and J. H. Whitelaw (Springer-Verlag, Berlin, Heidelberg, 1985).

${ }^{57}$ S. B. Pope, Turbulent Flows (Cambridge University Press, Cambridge, 2000).

${ }^{58}$ Y. Zhou, A. S. Wexler, and L.-P. Wang, "On the collision rate of small particles in isotropic turbulence. II. Finite inertia case," Phys. Fluids 10, 1206 (1998).

${ }^{59}$ S. Stolz, N. A. Adams, and L. Kleiser, "An approximate deconvolution model for large-eddy simulation with application to incompressible wall-bounded flows," Phys. Fluids 13, 997 (2001).

${ }^{60}$ J. C. H. Fung, J. C. R. Hunt, N. A. Malik, and R. J. Perkins, "Kinematic simulation of homogeneous turbulence by unsteady random Fourier modes," J. Fluid Mech. 236, 281-318 (1992).

${ }^{61}$ N. A. Malik and J. C. Vassilicos, "A Lagrangian model for turbulent dispersion with turbulent-like flow structure: Comparison with direct numerical simulation for two-particle statistics," Phys. Fluids 11, 1572 (1999).

${ }^{62} \mathrm{P}$. Flohr and J. C. Vassilicos, "A scalar subgrid model with flow structure for large-eddy simulations of scalar variances," J. Fluid Mech. 407, 315-349 (2000).

${ }^{63}$ J. Jeong and F. Hussain, "On the identification of a vortex," J. Fluid Mech. 285, 69-94 (1995).

${ }^{64}$ M. R. Maxey, "The gravitational settling of aerosol particles in homogeneous turbulence and random flow fields," J. Fluid Mech. 174, 441-465 (1987).

${ }^{65}$ H. M. Blackburn, N. N. Mansour, and B. J. Cantwell, "Topology of fine-scale motions in turbulent channel flow," J. Fluid Mech. 310, 269-292 (1996).

${ }^{66} \mathrm{C}$. Nilsen and H. I. Andersson, "Mechanisms of particle clustering in Gaussian and non-Gaussian synthetic turbulence," Phys. Rev. E 90, 043005 (2014). 